\title{
Laser Tweezers: A tool to assist self-assembly and template population
}

\author{
By
}

\author{
Xue Fei Pan
}

\begin{abstract}
A thesis submitted to the
Faculty of Graduate Studies and Research

In partial fulfillment of the requirements

For the degree of

Master of Applied Science
\end{abstract}

Ottawa-Carleton Institute for Electrical Engineering,

Department of Electronics,

Carleton University,

Ottawa, Ontario, Canada

January 2005

(C) Xue Fei Pan 2005 


$\begin{array}{ll}\begin{array}{l}\text { Library and } \\ \text { Archives Canada }\end{array} & \begin{array}{l}\text { Bibliothèque et } \\ \text { Archives Canada }\end{array} \\ \begin{array}{l}\text { Published Heritage } \\ \text { Branch }\end{array} & \begin{array}{l}\text { Direction du } \\ \text { Patrimoine de l'édition }\end{array} \\ \begin{array}{l}\text { 395 Wellington Street } \\ \text { Ottawa ON K1A ON4 }\end{array} & \begin{array}{l}\text { 395, rue Wellington } \\ \text { Ottawa ON K1A ON4 } \\ \text { Canada }\end{array}\end{array}$

Your file Votre référence

ISBN: 0-494-00752-4

Ourfile Notre référence

ISBN: 0-494-00752-4

NOTICE:

The author has granted a nonexclusive license allowing Library and Archives Canada to reproduce, publish, archive, preserve, conserve, communicate to the public by telecommunication or on the Internet, loan, distribute and sell theses worldwide, for commercial or noncommercial purposes, in microform, paper, electronic and/or any other formats.

The author retains copyright ownership and moral rights in this thesis. Neither the thesis nor substantial extracts from it may be printed or otherwise reproduced without the author's permission.
AVIS:

L'auteur a accordé une licence non exclusive permettant à la Bibliothèque et Archives Canada de reproduire, publier, archiver, sauvegarder, conserver, transmettre au public par télécommunication ou par l'Internet, prêter, distribuer et vendre des thèses partout dans le monde, à des fins commerciales ou autres, sur support microforme, papier, électronique et/ou autres formats.

L'auteur conserve la propriété du droit d'auteur et des droits moraux qui protège cette thèse. $\mathrm{Ni}$ la thèse ni des extraits substantiels de celle-ci ne doivent être imprimés ou autrement reproduits sans son autorisation.
In compliance with the Canadian

Privacy Act some supporting forms may have been removed from this thesis.

While these forms may be included in the document page count, their removal does not represent any loss of content from the thesis.
Conformément à la loi canadienne sur la protection de la vie privée, quelques formulaires secondaires ont été enlevés de cette thèse.

Bien que ces formulaires aient inclus dans la pagination, il n'y aura aucun contenu manquant.

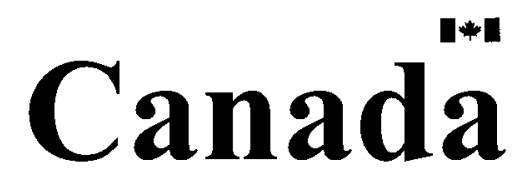




\begin{abstract}
Photonic crystals and photonic quasi-crystals as novel materials have the potential to stimulate the development of optical systems in the $21^{S T}$ century. Similar to electrons, which have a band gap over a range of frequencies in the semiconductors, photons also have a band gap over a region of optical frequencies in photonic crystals.

Self-organization of colloidal photonic crystals as a fast and simple fabrication method has been widely used and researched in microelectronics and optics. However, the presence of defects such as array shift and vacant sites in colloidal micro-spheres results in disorder and reduction of the band gap in photonic crystals. This thesis investigates the use of laser tweezers to manipulate micron-size polystyrene colloidal particles. In order to control the 2D monolayer photonic crystals and quasi-crystals during the self-organization process, laser tweezers are used to minimize defects and pattern to templates. The average defect density can be reduced from $0.0047 \mu \mathrm{m}^{-2}$ to $0.0022 \mu \mathrm{m}^{-2}$ for $2 \mathrm{D}$ photonic crystals. And the average defect density can be obtained as low as $0.00060 \mu \mathrm{m}^{-2}$ for colloidal quasi-crystals. In this thesis, laser tweezers are investigated to determine whether this instrument can be used to control the organization of colloidal polystyrene particles in 2D arrays and on surface layer templates. The colloidal modeling systems set up in this thesis using laser tweezers provide a useful tool in both the study of colloidal crystallization and the processing of new structures in optics and microelectronics.
\end{abstract}




\section{Acknowledgements}

I would first like to thank Professor Robert Gauthier of Carleton University for his invaluable guidance and advice throughout this research. I thank him for providing research direction, insights, ideas and encouragement. He helped me to make the transition from twelve years of full time employment to academic life and made it interesting, challenging and fun.

I would also like to thank Professor Langis Roy and Department of Electronics staff members Betty Zahalan and Peggy Piccolo for their administrative help. For their friendship and assistance, I also thank fellow graduate students in the Department of Electronics.

This thesis would not have been possible without the support and encouragement of my family. Thanks to my wife, Yan Li Qu, who after the initial shock, pushed, prodded and praised me through all the hard work; and finally my daughter, Ying He Pan, who had to put up with a Dad who had more homework than she did. Thank you very much for all your support. 


\section{Table of Contents}

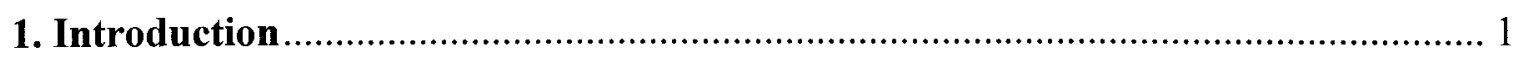

1.1 Background and Motivation .............................................................................. 1

1.2 Objective and Contributions .......................................................................... 2

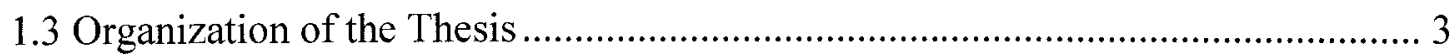

2. Photonic Crystals and Colloidal Crystallization .................................................. 4

2.1 Photonic Band Gap Crystals ............................................................................... 4

2.2 Maxwell's Equation in Photonic Crystals............................................................. 6

2.3 Photonic Band Gap ……………….............................................................. 7

2.3.1 The Origin of Photonic Band Gap ……………………............................... 7

2.3.2 Photonic Crystals Based on Inverted Opals.................................................... 9

2.4 Defects in Photonic Crystals ........................................................................... 11

2.5 Photonic Quasi-Crystals................................................................................. 13

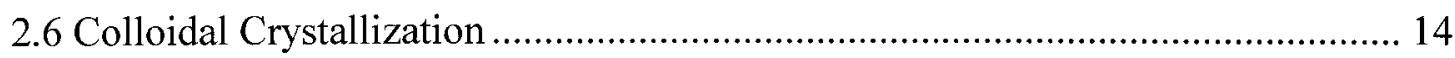

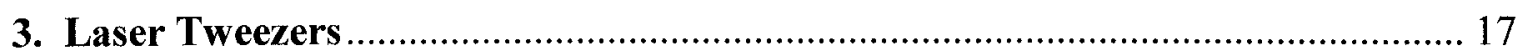

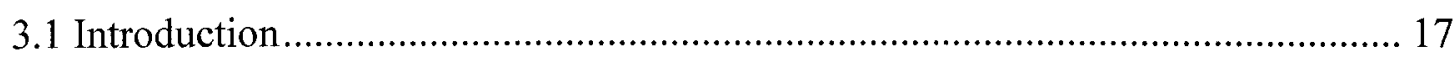

3.2 Fundamentals of Light Propagation............................................................. 22

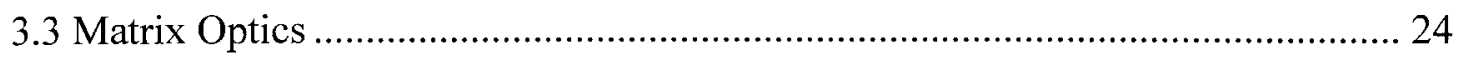

3.4 Gaussian Beam Model ................................................................................... 30 


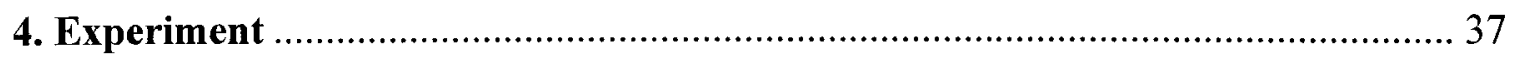

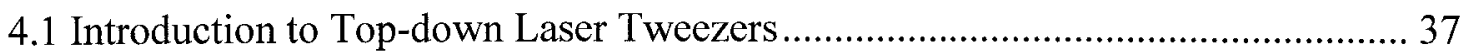

4.2 Equipment Used in Laser Tweezers ..................................................................... 39

4.3 The Laser Beam Delivery System Setup ………………..................................... 45

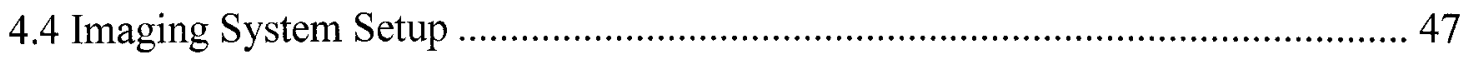

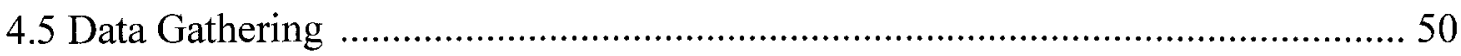

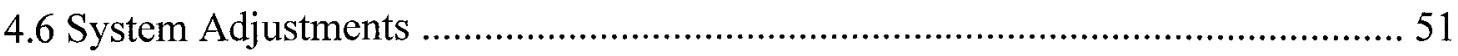

4.7 Manipulation of Colloidal Particles in Chamber .................................................... 53

4.8 Preparation of Patterned Template ........................................................................ 54

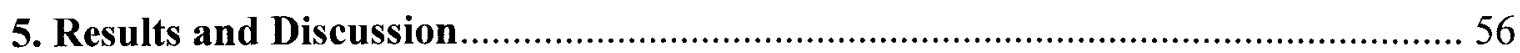

5.1 Assembly of Colloidal Crystals with Non-Template............................................. 56

5.1.1 Proof of Laser Trapping and Manipulation ……………………………...... 56

5.1.2 Assembly Using Laser Tweezers.............................................................. 57

5.1.3 Results and Discussion of Assembly of 2D Colloidal Crystals .................... 58

5.2 Assembly of 2D Colloidal Crystals with Template .................................................. 66

5.2.1 Assembly of 2D Colloidal Crystals with Cylindrical Cavity Template ...... 66

5.2.2 Assembly of 2D Photonic Crystals with Grid Template ………………...... 69

5.3 Assembly of Quasi-Crystals with Template .......................................................... 73

5.3.1 Results................................................................................................. 73

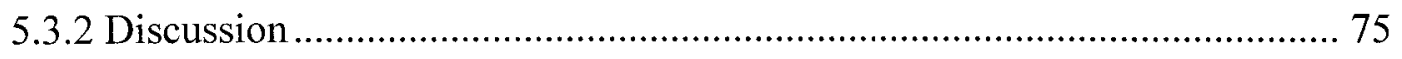


6. Conclusion and Recommendations

References

Appendix 


\section{List of Figures}

Figure 2.1 Schematic illustration of one-dimensional (1D), two-dimensional (2D), and three-dimensional (3D) photonic crystals .......................................... 5

Figure 2.2 2D colloidal crystals face-center cubic lattice structure fabricated

with polystyrene latex particles .............................................................. 5

Figure 2.3 a. FCC lattice structure. b. Brillouin zone of an FCC lattice structure.......... 10

Figure 2.4 Band structure for a close-packed FCC lattice of

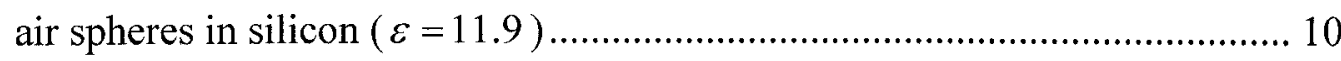

Figure 2.5 DOS for a close-packed FCC lattice of air spheres in silicon $(\varepsilon=11.9) \ldots \ldots . .11$

Figure 2.6 2D colloidal crystals face-center cubic lattice structure fabricated with

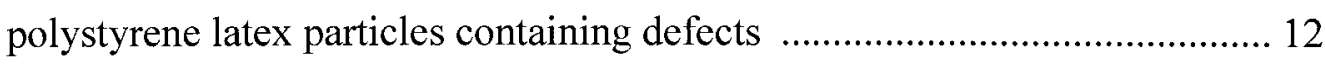

Figure 2.7 8-fold and 12-fold quasi-crystal pattern embedded in the silicon................. 13

Figure 2.8 Three stages of colloidal crystallization .................................................. 15

Figure 3.1 a. Illustration of laser tweezers acting on the particle within the focused spot. b. An actual laser tweezers spot image taken from the experiment................ 18

Figure 3.2 Schematic of resultant force directed toward the focus of the laser beam ...... 21

Figure 3.3 Diagram of a simple optical imaging system ........................................ 25

Figure 3.4 Diagram of a simple optical imaging system using thin lens....................... 28

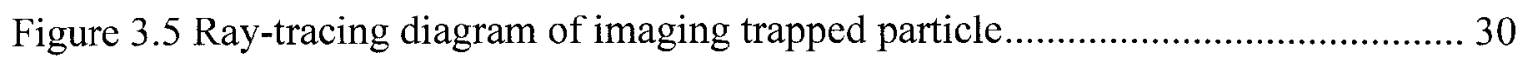

Figure 3.6 The Rayleigh range and depth of focus of a Gaussian beam ....................... 31

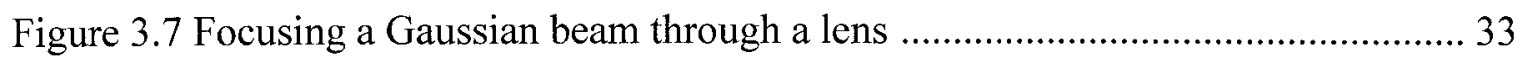


Figure 3.8 Focusing a Gaussian beam through a lens................................................. 35

Figure 4.1 Schematic of the top-down laser tweezers experimental setup ....................... 38

Figure 4.2 Major components shown in the top-down laser tweezers setup .................... 39

Figure 4.3 Laser diode drivers (LDC 500), the DB9 interface cable and

laser diode $\quad(\lambda=785 \mathrm{~nm}, P=70 \mathrm{~mW}, I=100 \mathrm{~mA})$................................. 40

Figure 4.4 XY translator, aspheric lens adaptor, laser diode mount and $\mathrm{Z}$ translator ...... 40

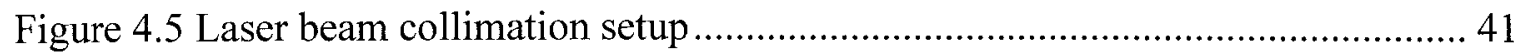

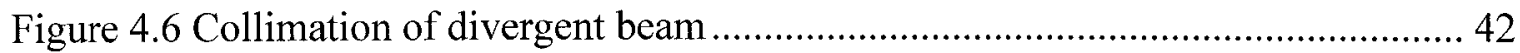

Figure 4.7 Aspheric lens and objective lens ..................................................................... 42

Figure 4.8 XYZ translation stage and CCD camera ........................................................ 43

Figure 4.9 Cage cube and kinematics mounting platform ................................................ 43

Figure 4.10 Beam splitter and filter ............................................................................ 44

Figure 4.11 IR viewing card and alignment tool for cage system ................................... 44

Figure 4.12 Laser beam delivery system of the top-down laser tweezers setup............... 46

Figure 4.13 Imaging system of the top-down laser tweezers setup ................................. 49

Figure 4.14 Schematic of data gathering ……………….......................................... 51

Figure 4.15 Experimental dual beams multiple exposure optical system.......................... 55

Figure 4.16 Photonic crystal pattern substrate (left) and

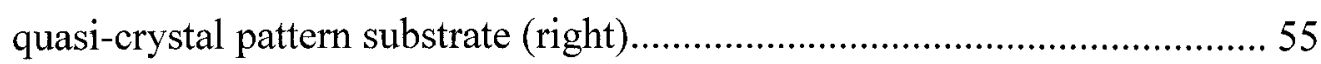

Figure 5.1 Illustration of particle displacement under the laser tweezers......................... 57

Figure 5.2 Process of forming the 2D monolayer of polystyrene particles with a diameter of $2 \mu m$ under the action of laser tweezers on a flat glass substrate. 58 
Figure 5.3 Optical microscopic images of the 2D colloidal crystal sample with an actual size of $82.0 \times 62.0 \mu \mathrm{m}^{2}$ 61

Figure 5.4 Schematic representation of a droplet of a colloidal suspension drying slowly on flat wall 63

Figure 5.5 Typical close-packed 2D colloidal

photonic crystals obtained using laser tweezers. 64

Figure 5.6 Scanning electron micrograph (SEM) of 2D monolayer

colloidal crystals with $2 \mu \mathrm{m}$ polystyrene particles using laser tweezers 66

Figure 5.7 Video images of 2D arrays of colloidal aggregates assembled under the confinement of temples etched in the photoresist using laser tweezers. 68

Figure 5.8 Process of self-organizing the 2D monolayer of polystyrene photonic crystals with a diameter of $2 \mu m$ under the action of laser tweezers on patterned template 70

Figure 5.9 Typical colloidal crystals based on the square 2D grating template .............. 71

Figure 5.10 a. Particle arrangement b. Lattice geometry. 72

Figure5.11 Process pictures showed the particles were close-packed together along the cavity of quasi-crystal pattern when the water evaporated. 74

Figure5.12 Optical microscopic images of the 2D quasi-crystal sample with an actual size of $82.0 \times 62.0 \mu \mathrm{m}^{2}$ 75 


\section{List of Tables}

Table 3.1 Effective Focal Lengths for Typical Lenses .......................................... 29

Table 3.2 Actual Image Sizes for Typical Objective Lenses................................... 29

Table 3.3 The Beam Waist Radius and Depth of Focus for Three Typical Lenses ....... 36

Table 5.1 Statistical Results of 2D Monolayer Colloidal Crystals for Fig.5.2 ….......... 62

Table 5.2 Typical Statistical Results of 2D Monolayer Colloidal Crystals for Fig.5.6... 65

Table 5.3 Control of the Self-Assembled Particles under Cylindrical Cavity Templates 67

Table 5.4 Typical Statistical Results of 2D Colloidal Quasi-Crystals for Fig.5.13 ....... 76 


\section{List of Symbols}

$\Gamma \quad$ The center of the Brillouin zone of the FCC lattice structure

$\varepsilon_{b} \quad$ The dielectric constant for scattering materials such as spheres

$\rho \quad$ The radial distance

$\mu_{0} \quad$ The permeability of a vacuum

$\varepsilon(r) \quad$ The relative permittivity

$\mu(r) \quad$ The relative permeability

$\omega \quad$ Angular frequency

$\omega_{n}(\vec{k}) \quad$ Eigenfrequencies of photonic crystals

$\tilde{\omega} \quad$ Center frequency of photonic band gap

$\lambda \quad$ Wavelength

$\vec{B}(r, t) \quad$ Magnetic flux density

$c \quad$ The velocity of light in a vacuum

$\vec{D}(r, t) \quad$ Electric flux density

$\vec{E}(r, t) \quad$ Electric field strength

$E_{s} \quad$ The electric field of a Gaussian beam

$f \quad$ The focus length of the lens

$F_{\text {grad }} \quad$ Gradient forces

$F_{\text {scat }} \quad$ Scattering forces

$H(\vec{r}) \quad$ The $\mathrm{z}$ component of the magnetic field

$\vec{H}(r, t) \quad$ Magnetic field strength, 
$I_{s} \quad$ The intensity of a Gaussian beam

$\vec{J}(r, t)$ Electric current density

$n_{1} \quad$ The refractive index of water

$n_{2} \quad$ The refractive index of glass

$n_{3} \quad$ The refractive index of air

$n_{a} \quad$ The refractive index of suspension solvent

$n_{b} \quad$ The refractive index of a spherical particle

$\overrightarrow{R_{i}} \quad$ Real space lattice vectors

$\vec{k} \quad$ Wave vector

$k \quad$ Wave number

$L \quad$ The center of the hexagonal faces of a Brillouin zone (FCC lattice structure)

$r_{1} \quad$ The curvature of the lens at left side

$r_{2} \quad$ The curvature of the lens at right side

$R_{e} \quad$ Fresnel reflection coefficient

$R_{\text {ave }} \quad$ The average Fresnel reflection coefficient

$R_{e p} \quad$ The reflection coefficient for light polarized vertical to the plane of incidence

$R_{e s} \quad$ The reflection coefficient for polarization parallel to the plane of incidence

$R(r) \quad$ The spherical refraction matrix

$R(z) \quad$ The wavefront radius of curvature

$T \quad$ The Fresnel transmitted coefficient

$T_{\text {ave }} \quad$ The average Fresnel transmitted coefficient

$T_{p} \quad$ The transmitted coefficient for light polarized vertical to the plane of incidence 
$T_{s} \quad$ The transmitted coefficient for polarization parallel to the plane of incidence

$T(d) \quad$ The translation matrix

$X \quad$ The center of the square faces of a Brillouin zone (FCC lattice structure)

$W_{0} \quad$ The Gaussian beam waist radius

$W(z) \quad$ The beam width

$z_{0} \quad$ Rayleigh range 


\section{Chapter 1}

\section{Introduction}

\subsection{Background and Motivation}

It is anticipated that the next-generation of computers may process information at the speed of light. What kind of material can give us complete control over light propagation in these computer chips while making them smaller, faster, cheaper and more energy efficient?

Over the past decade, material research has produced a tremendous variety of synthetic structures. As a result, artificial materials showing suitable mechanical, chemical, electronic and optical properties can now be produced. Another factor spurring new material research is that present telecom systems contain an awkward mixture of optics and electronics. While present switching and routing systems, as well as the "last mile" to the customer, still depend on slower electronic components, purely optical systems would permit the very high-speed data rates required by the Internet.

The work of Yablanovitch on possible ways of inhibiting spontaneous emission [1] and Sajeev John on localization of light in strongly scattering dielectric structures [2] resulted in a new kind of artificial structured material in which light (photons) behaves similarly to electrons in semiconductors. The basic idea is to design dielectric materials whose optical band gap will forbid electromagnetic wave propagation much like ordinary semiconductor crystals forbid electrons with momentum in the gap. In a photonic crystal, the periodic dielectric structure material is analogous to a periodic potential. The extent 
of the photonic band gap is related to the dielectric constant of the periodic material. The possibility exists of controlling photons and creating photonic band gap artificial materials, resulting in a systematic method for molding the propagation properties of electromagnetic waves [3].

Among the many fabrication techniques of photonic crystals, the selforganization of colloidal particles is a controlled approach used to form $2 \mathrm{D}$ and $3 \mathrm{D}$ periodic structures [4]-[6]. Mono-disperse submicron-sized spheres of either silica or polystyrene are suitable for producing templates from which inverse opals of appropriate dielectric profile can exhibit complete photonic band gap [7]. Defects, array shifts and vacancies introduced in the growth of the template may reduce the band gap [8]. In this thesis, laser tweezers are investigated to determine whether this instrument can be used to control the organization of colloidal polystyrene particles in $2 \mathrm{D}$ arrays and on surface layer templates.

\subsection{Objective and Contributions}

The objective of this thesis is to apply a self-organization fabrication technique through a single beam laser trapping method to control the formation of desired $2 \mathrm{D}$ colloidal crystals on different kinds of surfaces. The main contributions made by this thesis are:

1. Using a self-organization method via laser tweezers to grow $2 \mathrm{D}$ one layer photonic crystals on a flat surface.

2. Using a self-organization method via laser tweezers to grow $2 \mathrm{D}$ one layer photonic crystals on a patterned template. 
3. Using a self-organization method via laser tweezers to grow $2 \mathrm{D}$ colloidal crystals on a quasi-crystal template.

\subsection{Organization of the Thesis}

Chapter two describes the theory related to photonic band gap crystals and the colloidal fabrication method using self-organization. Chapter three describes the theory of optical laser tweezer systems. In Chapter four, the experimental method and instrumental design related to the Top-down version of a laser tweezer system are introduced. Experimental results related to assembly control and template population are presented in Chapter five. Conclusions and recommendations for future work are provided in Chapter six. 


\section{Chapter 2}

\section{Photonic Crystals and Colloidal Crystallization}

\subsection{Photonic Band Gap Crystals}

Photonic crystals are periodic arrays of materials with different refractive indices. The spatial period of different materials is called the lattice constant and is equivalent to the lattice constant of ordinary crystals composed of a periodic array of atoms. In this way, the fundamental theories of photonic crystals are closely related to the theory regarding atom level crystals. An important difference between photonic crystals and ordinary crystals is the scale of the lattice constant. Photonic crystals are on the order of the wavelength of the relevant electromagnetic waves, while ordinary crystals are on the order of angstroms.

Photonic crystals are classified mainly into three categories: one-dimensional (1D), two-dimensional (2D), and three-dimensional (3D), based on the dimensionality of the stack. Figure 2.1 shows the simplest case in which two materials denoted by A and B are stacked alternately. 


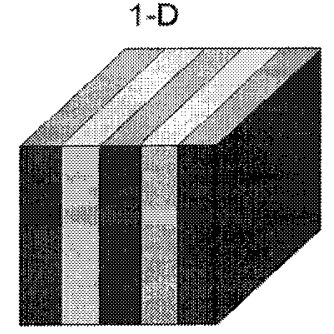
periodic in
one ditection

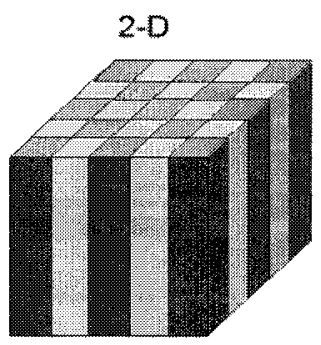

periodic in wo directions

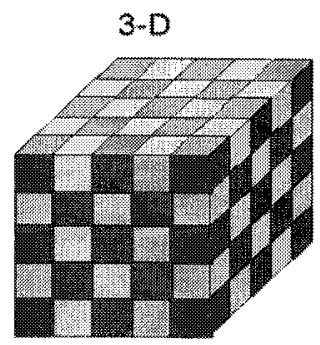

periodic in
hree direction

Figure 2.1 Schematic illustrations of one-dimensional (1D), twodimensional (2D), and three-dimensional (3D) photonic crystals [9].

In recent years, most investigation of photonic crystals has concentrated on 2D and $3 \mathrm{D}$ crystals. Figure 2.2 shows the $2 \mathrm{D}$ colloidal crystals formed by polystyrene particles, without mismatch in the crystal lattice, fabricated in our optics lab. The bright spots are the spheres, which are arranged in a hexagonal stack.

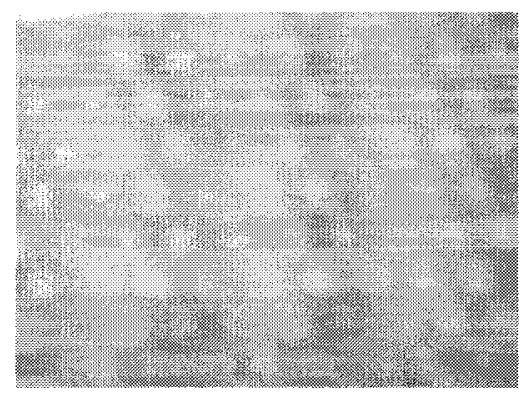

Figure 2.2 2D colloidal crystals face-center cubic lattice structure fabricated with polystyrene latex particles. 


\subsection{Maxwell's Equations in Photonic Crystals}

The propagation of light waves in photonic crystal is governed by Maxwell's equations [8]. These equations can be expressed as follows:

$$
\begin{gathered}
\nabla \cdot \vec{D}(\vec{r}, t)=\rho(\vec{r}, t) \\
\nabla \cdot \vec{B}(\vec{r}, t)=0 \\
\nabla \times \vec{E}(\vec{r}, t)+\frac{\partial}{\partial t} \vec{B}(\vec{r}, t)=0 \\
\nabla \times \vec{H}(\vec{r}, t)-\frac{\partial}{\partial t} \vec{D}(\vec{r}, t)=\vec{J}(\vec{r}, t)
\end{gathered}
$$

where $\vec{E}(\vec{r}, t)$ is the electric field strength, $\vec{H}(\vec{r}, t)$ is the magnetic field strength, $\vec{D}(\vec{r}, t)$ is the electric flux density, $\vec{B}(\vec{r}, t)$ is the magnetic flux density, $\vec{J}(\vec{r}, t)$ is the electric current density and $\rho(\vec{r}, t)$ is the electric charge density.

When photonic crystals are created using material that is isotropic and low-loss, this implies that $\vec{D}(\vec{r}, t)=\varepsilon(\vec{r})[\vec{E}(\vec{r}, t)]$, where $\varepsilon(\vec{r})$ is the relative permittivity and is approximated as real and constant. Similarly, with respect to the magnetic flux density and magnetic field, the relationship is $\vec{B}(\vec{r}, t)=\mu(\vec{r}) \vec{H}(\vec{r}, t)$. For the majority of dielectric materials, the relative permeability is very close to unity, so that $\vec{B}(\vec{r}, t)=\mu_{0} \vec{H}(\vec{r}, t)$. There are no free charges or currents; therefore $\vec{J}=\rho=0$. Using these observations and time-harmonic fields with angular frequency $\omega$, the final expression in terms of $\vec{H}(\vec{r})$ is:

$$
\nabla \times\left(\frac{1}{\varepsilon(\vec{r})} \nabla \times \vec{H}(\vec{r})\right)=\left(\frac{\omega}{c}\right)^{2} \vec{H}(\vec{r})
$$

Equation (2.5) can be cast as an eigenvalue problem for a given photonic crystal. 


\subsection{Photonic Band Gap}

\subsubsection{The Origin of Photonic Band Gap}

The following is a summary of how photonic band gap comes about, as presented by Johnson and Joannopuulos [9]. Equation (2.5) is a Hermitian eigenproblem over an infinite domain, and generally produces a continuous spectrum of eigenfrequencies $\omega$.

From the Bloch-Floquet theorem, for a Hermitian eigenproblem whose operators are periodic functions of position, the solutions can always be chosen of the form $e^{i \vec{k} \cdot \vec{x}} \cdot f(\vec{x})$. A periodic function $f(\vec{x})$ is $f\left(\vec{x}+\overrightarrow{R_{i}}\right)=f(\vec{x})$ for any $\vec{x}$ and any primitive lattice vector $\vec{R}_{i}$ of the crystal. A corollary of this theorem is that the wave vector $\vec{k}$ is a conserved quantity, and hence propagating waves with a fixed $\vec{k}$ cannot scatter.

Applying the Bloch-Floquet theorem to Eq (2.5), the solution of Eq (2.5) for a periodic permittivity $\varepsilon$ can be chosen of the form:

$$
\vec{H}(\vec{r})=e^{i(\vec{k} \cdot \vec{x})} \overrightarrow{H_{\vec{k}}}
$$

where $\overrightarrow{H_{\vec{k}}}$ is a periodic function of position. Substituting Eq. (2.6) into Eq. (2.5), $\overrightarrow{H_{\vec{k}}}$ satisfies the following Hermitian eigenproblem [9]:

$$
(\nabla+i \vec{k}) \times \frac{1}{\varepsilon}(\nabla+i \vec{k}) \times \overrightarrow{H_{\vec{k}}}=\left(\frac{\omega}{c}\right)^{2} \overrightarrow{H_{\vec{k}}}
$$

Eigenproblems with a finite domain have a discrete set of eigenvalues. Equation (2.7) can be solved over a finite domain because of periodic function $\overrightarrow{H_{\vec{k}}}$. The eigenfrequencies $\omega$ are a countable discrete sequence of continuous functions: $\omega_{n}(\vec{k})$ (for $n=1,2,3, \ldots$ ). When they are plotted as a function of the wave vector $\vec{k}$, these frequencies form the band structure of the crystal. 
There are two general properties of Hermitian eigenvalue problems. First, the eigenfunctions must be orthogonal [9], so that

$$
\int \vec{H}_{\vec{k}}^{(m)^{*}} \cdot \vec{H}_{\vec{k}}^{(n)}=0
$$

for any two eigenstates $(m \neq n)$ at a given $\vec{k}$ point. Second, the lowest band solves a variational problem [9]:

$$
\omega_{1}(\vec{k})^{2}=\frac{\min }{\vec{H}_{\vec{k}}} \frac{\int\left|(\vec{\nabla}+i \vec{k}) \times \vec{H}_{\vec{k}}\right|^{2} / \varepsilon}{\int\left|\vec{H}_{\vec{k}}\right|^{2}}
$$

The second band meets the same variational problems, with the added constraint that it be orthogonal to the first band according to Eq. (2.8), and similarly for the third band and so on. The following properties help to minimize the variational problem of Eq. (2.9): first, the curl should not be too large, should vary slowly, and not contain any fast oscillations; second, the curl should be concentrated in regions of high dielectric constant $\varepsilon$. Similarly, for the second band, the curl also needs to oscillate slowly and be concentrated in the high dielectric regions. However, the second eigenfunction must also be orthogonal to the first band from Eq. (2.8). In order to satisfy this, the second band must be concentrated in lower dielectric to make the integral zero as expressed in Eq. (2.8). The consequence is a large increase in frequency in Eq. (2.9), thus creating a band gap. In order to have an omni-directional band gap, the gaps at all $\vec{k}$ points must overlap in some frequency range. The details of the dispersion relation $\omega_{n}(\vec{k})$ can not be probed in the transmission experiments on PBG materials. Instead, the number of states can be measured for a given 
direction of propagation. The total DOS (Density of States) could be obtained by integrating the number of states with all directions. This describes the average behavior of the structure. The DOS is another important quantity characterizing the photonic crystal. In a photonic crystal, the DOS presents jumps with peaks and dips around a mean value given by the effective medium theory.

\subsubsection{Photonic Crystals Based on Inverted Opals}

The inverted opal packed FCC lattice consists of air spheres of a dielectric constant $\varepsilon_{\text {air }}=1$ immersed in a dielectric matrix $\left(\varepsilon_{b}\right)$. The FCC lattice structure and the Brillouin zone of an FCC lattice structure are shown in Figure 2.3. This is a diamond-like structure, and has fourteen faces. There are 8 hexagonal faces and 6 square faces. Some key points are: $[\Gamma:(0,0,0)]$, the center of the FCC structure, $\left[L:\left(\frac{\pi}{a}, \frac{\pi}{a}, \frac{\pi}{a}\right)\right]$, the center of the hexagonal faces, and $\left[X:\left(\frac{2 \pi}{a}, 0,0\right)\right]$, the center of the square faces.

Figure 2.4 shows the band structure and Figure 2.5 shows the corresponding total DOS of an inverted FCC structure consisting of close-packed air spheres in a silicon matrix with dielectric constant $\varepsilon_{b} \approx 11.9$. From the drawing, we can see that the FCC structure possesses a pseudo-gap between the fourth and fifth bands around $\widetilde{\omega}=0.524$ and a complete $4.25 \%$ band gap between the eighth and ninth bands with a center frequency of $\tilde{\omega} \approx 0.794[10]$. 


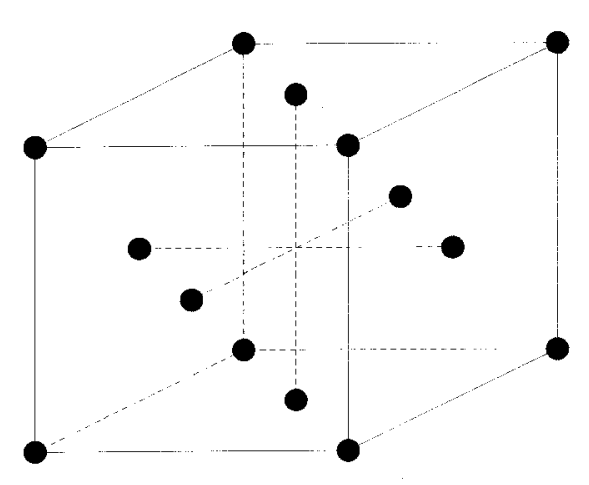

a.

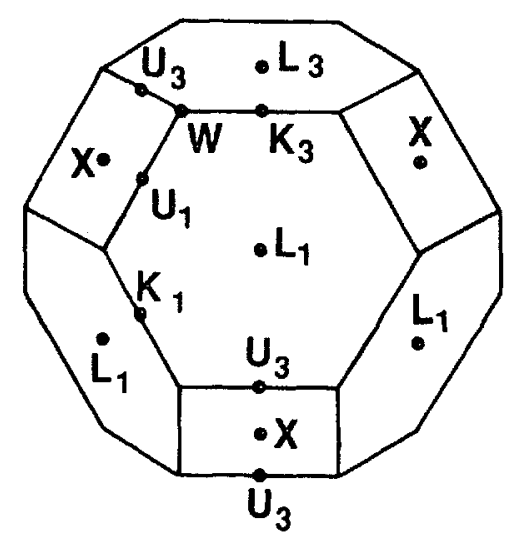

b.

Figure 2.3 a. FCC lattice structure. b. Brillouin zone of an FCC lattice structure.

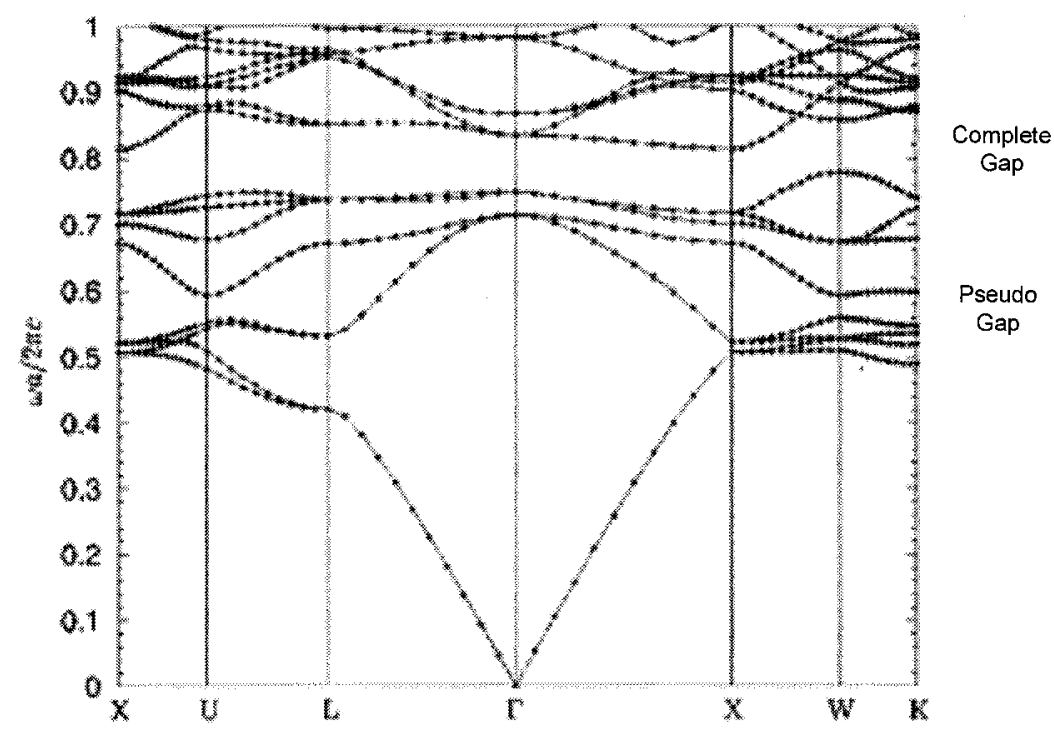

Figure 2.4 Band structure for a close-packed FCC lattice of air spheres in silicon $(\varepsilon=11.9)[10]$. 


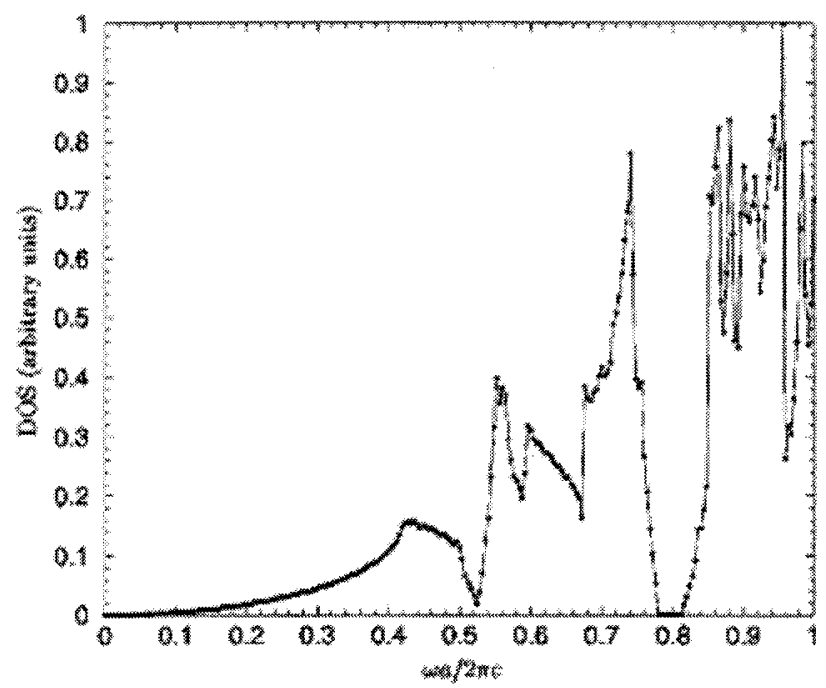

Figure 2.5 DOS for a close-packed FCC lattice of air spheres in silicon $(\varepsilon=11.9)$ [10].

\subsection{Defects in Photonic Crystals}

Defects are local variations in the lattice structure of a photonic crystal. These undesirable variations can cause diffraction losses in the propagating fields, destroying the photonic confinement capability. The band gap may be reduced significantly and even close at a fluctuation magnitude as small as $2 \%$ of the lattice constant [11]. A typical inverted opal crystal can be prepared as follows. First, one should have a template assembled from a self-organizing system, using, for instance, monodisperse silica or polystyrene spheres. Then, matrix material is used to backfill the void space between spheres by sintering. Finally, the inverse-opal crystal is obtained by selectively etching the colloidal spheres. In practice, defects can occur at every step of the fabrication process. The spheres may fail to array at the lattice sites, causing planar stacking faults and vacant sites. 
The 2D monolayer colloidal crystals shown in Figure 2.6 are obtained by the selfassembly method. From the picture, we can see that the defective portion (array shift and vacant sites) causes variations in the lattice structure of the $2 \mathrm{D}$ colloidal crystals. When counting the number of the defects within a specific area, two kinds of defects are included: the first is line type, corresponding to the crystal array shift along a line, and the second is point type, which is usually due to vacancy. The minimization of defects in the monolayer colloidal crystal is investigated using laser tweezers and templates to direct the growth of 2D monolayer colloidal crystal.

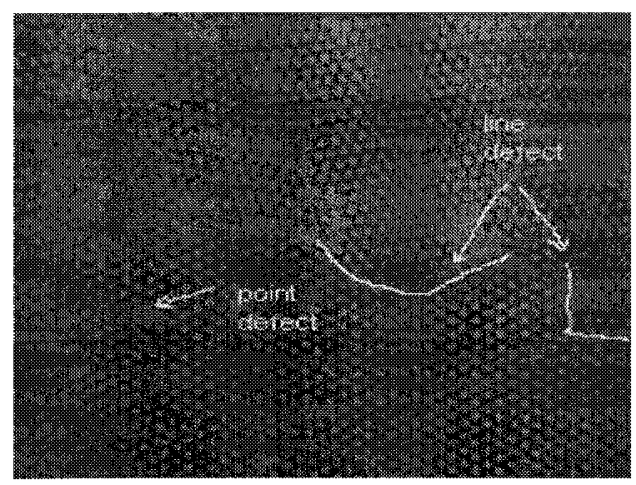

Figure 2.6 2D colloidal crystals face-center cubic lattice structure fabricated with polystyrene latex particles containing defects. 


\subsection{Photonic Quasi-Crystals}

In order to achieve complete and absolute photonic band gap from photonic crystals, the dielectric contrast must be increased and the structure must show translational symmetry. Another way to obtain the gap would be to use artificial quasicrystalline materials possessing multi-fold rotational symmetry. The high degree of rotational symmetry achievable in quasi-crystal patterns would lessen the high index contrast requirement for photonic crystals. The quasi-crystals possess $\mathrm{N}$-fold symmetry about a central feature and no long-range translational periodicity. The quasi-crystal pattern templates used in this work were fabricated in the laboratory and served as 2D patterned templates for growth and self-organizing. Two quasi-crystal patterns are shown in Figure 2.7 below [12]. The left figure shows an 8 -fold translational symmetry quasicrystal and the right figure shows a 12-fold translational symmetry quasi-crystal embedded in the silicon.
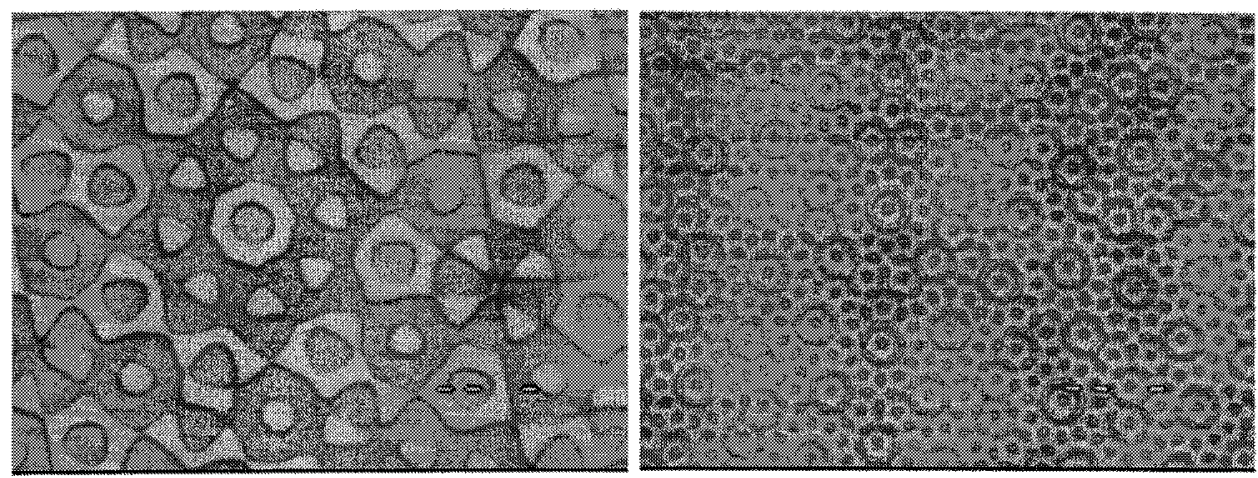

Figure 2.78 -fold and 12-fold quasi-crystal pattern embedded in the silicon [12]. 


\subsection{Colloidal Crystallization}

A colloidal particle is a macroscopic entity composed of a large number of atoms or molecules. The typical size of a colloidal particle ranges from a few nanometers to several micrometers. Colloidal epitaxial crystal growth is influenced by the underlying substrate formed with the geometry and periodicity of the lattice structures. The Selforganization of colloidal particles is an important technique used to assemble colloids during the drying of colloidal suspensions [13] [14].

The three stage model for the film formation was proposed by Vanderholl (1973) and Poehlein (1975) [15]. Figure 2.8 illustrates the three stages of colloidal crystallization.

Stage I: Water Evaporation and Particle Arrangement

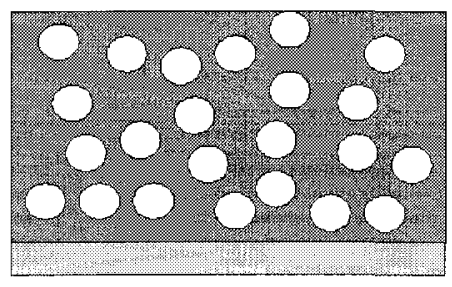

(a) Aqueous Dispersion:

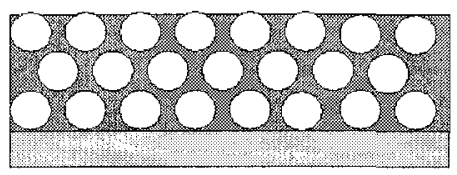

(b) Water Evaporation and Particle Arrangement

Stage II: Deformation 


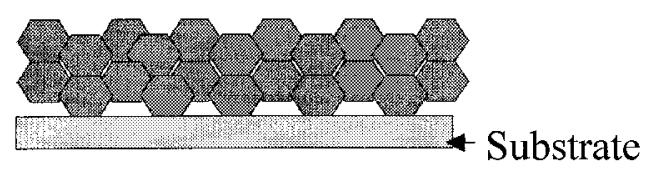

Stage III: Coalescence and Inter-Diffusion

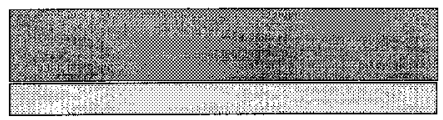

Figure 2.8 Three stages of colloidal crystallization.

\section{Stage I:}

Water evaporation occurs at a constant rate, and the solid concentration increases at the same time. During this stage, the particles are in continuous Brownian motion and as the distance between particles decreases, the electrostatic interactions (the screened Coulomb repulsion and the Van der Waals attraction) dominate over the thermal interactions. This stage comes to an end once enough water has evaporated to selfarrange the particles into a close-packed array.

\section{Stage II:}

This begins when the particles come into close contact. The rate of drying in this stage is lowered because of the decreasing air/water interface area available for evaporation. The particles undergo deformation because of coalescence under the influence of capillary forces based on the drying condition. Polystyrene spheres are not deformed in this stage.

\section{Stage III:}


This is the polymer inter-diffusion stage, when particle boundaries cease to exist and the diffusion of polymer chains takes place. The drying rate during this stage decreases drastically due to diffusion controlled evaporation. This stage will be omitted for polystyrene colloidal crystals.

Using the three stages, the self-assembly method is used to arrange colloids. As a result, we can obtain the $2 \mathrm{D}$ colloidal monolayer photonic crystals either at the flat wall or the periodic grid template. We can even create 2D colloidal monolayer quasi-crystals with the assistance of a quasi-crystal template. This method offers high processing speed, and is based simply on the sedimentation of particles and the evaporation of the solvent. In the same time period, multilayered and large area colloidal crystals can be obtained. Many factors influence colloidal crystallization, such as surface topography, and the form of the colloidal crystals is not predictable. The self-assembly method lacks local control over a template. The single beam laser trap / laser tweezers is a promising tool that can manipulate micrometer-sized colloidal particles without touching them. In order to minimize defects in the self-assembly of colloidal crystals, a laser tweezers is introduced in the self-assembly process to assist the growth of $2 \mathrm{D}$ colloidal crystals. Laser tweezers are described further in the following chapter. 


\section{Chapter 3}

\section{Laser Tweezers}

\subsection{Introduction}

Prior to the 1960s, optics formed the basis of a relatively small industry involving rather sedate and mature topics such as optical instruments, cameras, microscopes, and scientific applications. With the invention of the solid-state (ruby) laser, the gas laser, and the semiconductor injection laser, lasers have found a host of important applications in various fields such as medicine, atomic physics research, entertainment, fiber-optic communications, and military systems.

In 1970, Ashkin et al. demonstrated that tightly focused laser beams could trap and manipulate micrometer-sized particles [16]. This type of radiation pressure effect has been termed optical levitation. In 1986, this same researcher and co-workers demonstrated the stable three-dimensional trapping of micrometer-sized objects in the minimum waist region of a highly focused laser beam, the laser tweezers [17]. Laser tweezers allow for the trapping of particles (such as glass beads, polystyrene beads and colloidal gold), and have become a versatile tool in biology, material science and physics. A few milliwatts of laser power focused with a microscope objective lens is sufficient to form a robust tweezers.

Micronmeter diameter polystyrene particles are increasingly used in many advanced materials and optical devices. Polystyrene particles of $3 \mu \mathrm{m}$ can be used to produce ordered $2 \mathrm{D}$ colloidal crystals using radiation pressure [18]. A $10 \mu \mathrm{m}$ polystyrene particle 
can be used as a ball lens to increase fiber-to-fiber coupling [19]. Polystyrene particles can be seen under illumination while being trapped using laser tweezers.

As the laser passes through the objective lens of the laser tweezers, it is focused to a diffraction limited spot. The objective lens is used for both trapping and viewing. Dielectric particles will be seen to be pulled toward the high focus of the laser beam while being pushed away from the focus in the direction of the incident light. The scattering force is proportional to the intensity of the beam and points in the direction of the incident beam propagation. The gradient force is dependent on the intensity gradient of the beam, and points in the direction of the intensity gradient towards the focus [20]. Figure $3.1 \mathrm{a}$. illustrates the laser tweezers acting on the particles, and Figure $3.1 \mathrm{~b}$. shows an actual laser tweezers spot image focused on a sphere at the bottom of chamber.

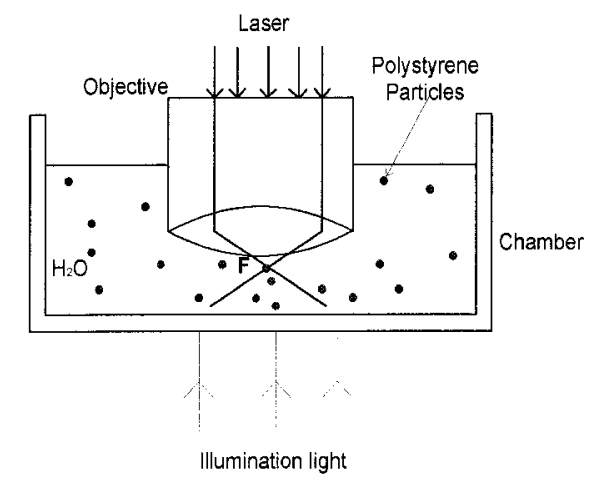

a.

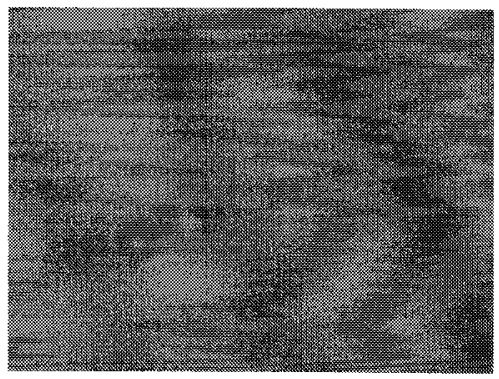

b.

Figure 3.1 a. Illustration of laser tweezers acting on the particles b. An actual laser tweezers spot image focusing at the bottom of chamber.

The Laser tweezers is a tool consisting of a single highly focused laser beam and 
microscopy apparatus. The basic principles related to the trapping and manipulation of micro-optic-particles is outlined as follows. The laser tweezers involves photons interacting with an object in the vicinity of a focused laser beam. The reflected rays tend to push the spheres along the beam and at the same time away from the high intensity beam region. The refracted rays pull the sphere into the axis of the beam and direct them toward the region where the beam is highly focused. The schematic of the trapping of a microsphere is illustrated in Figure 3.2. In Figure 3.2 (a), a typical pair of rays (a and b) of the converging beam are shown and focused above the center of micro-sphere. When they pass through the micro-sphere, they change direction due to refraction. The forces Fa and $\mathbf{F b}$ are shown pointing in the direction of the momentum change. The resultant force $\mathbf{F}=\mathbf{F a}+\mathbf{F b}$ is directed back to the focus and acts upwards on the micro-sphere. When using only two rays, the trap is stable when the center of the micro-sphere and the focus of the beam are in the same point. Similarly, in Figure $3.2(\mathrm{~b})$, when a pair of rays $a$ and $b$ are converging and focusing below the center of the micro-sphere, the resultant force $\mathbf{F}$ is directed back to the focus and acts downwards on the micro-sphere. Considering the use of two rays, the trap is stable when the center of the micro-sphere and the focus of beam are in the same point. In Figure 3.2 (c), when the pair of rays a and b are converging and focusing on the left side of the center of the micro-sphere, the resultant force $\mathbf{F}$ will be directed towards the beam axis. This brings the micro-sphere and beam central axis together. In Figure 3.2 (d), when the pair of rays $a$ and $b$ are converging and focusing at the right side of the center of the micro-sphere, the resultant force $\mathrm{F}$ is directed to the center of the micro-sphere and beam center of axis. 
In this two ray model, the sphere experiences an aligning and trapping force. The strength of the trap is determined by considering all rays incident onto the sphere. Stable traps are formed when the gradient force is stronger than the scattering force for spheres in the vicinity of the beam focus.

(a) Force up:

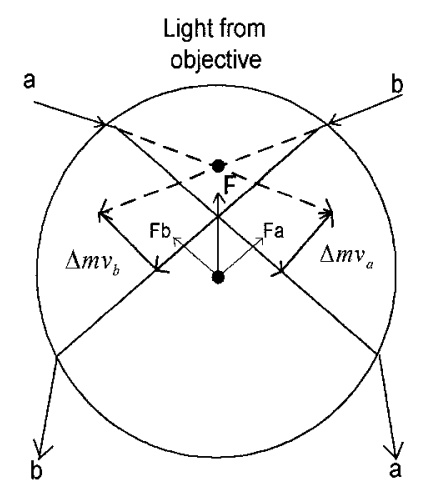

(b) Force down:

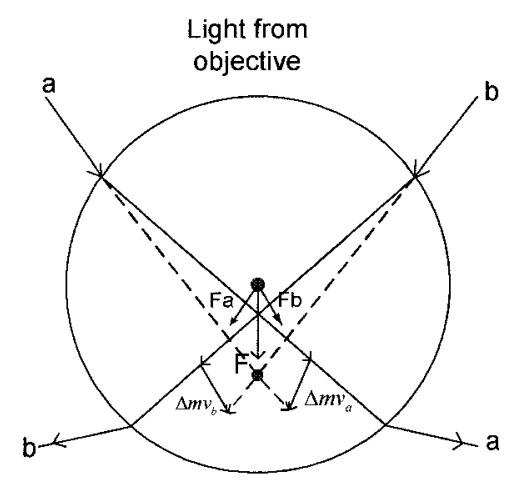

(c) Force left: 


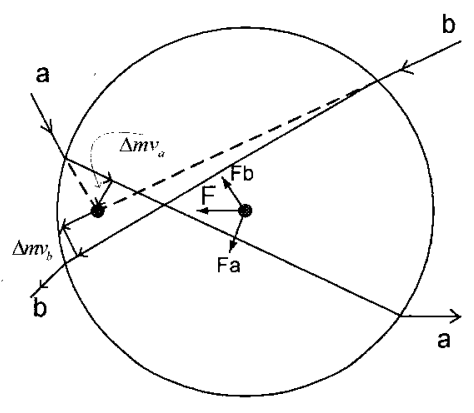

(d) Force right:

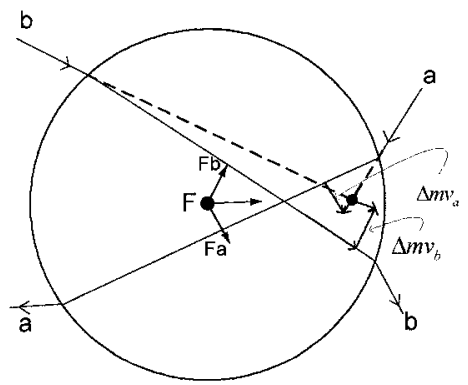

Figure 3.2 Schematic of resultant force directed toward the focus of the laser beam.

To explore the optical force and trapping, the optical force is usually decomposed into two vertical components. The gradient force component is directed toward the laser beam focus, while the scattering force is in the direction of the propagation of the beam. Ashkin gives the following equations of the scattering and gradient forces for an individual parallel incident ray on a sphere [20]:

$$
\begin{aligned}
& F_{\text {scat }}=\frac{n_{a} P}{c}\left\{1+R_{e} \cos \left(2 \theta_{1}\right)-\frac{T^{2}\left[\cos \left(2 \theta_{1}-2 \theta_{2}\right)+R_{e} \cos \left(2 \theta_{1}\right)\right]}{1+R_{e}^{2}+2 R_{e} \cos \left(2 \theta_{2}\right)}\right\} \\
& F_{\text {grad }}=\frac{n_{a} P}{c}\left\{R_{e} \cos \left(2 \theta_{1}\right)-\frac{T^{2}\left[\sin \left(2 \theta_{1}-2 \theta_{2}\right)+R_{e} \sin \left(2 \theta_{1}\right)\right]}{1+R_{e}{ }^{2}+2 R_{e} \cos \left(2 \theta_{2}\right)}\right\}
\end{aligned}
$$


where $P$ is the power of the incident ray, $\theta_{1}$ is the angle between the incoming beam and the normal to the sphere surface, $\theta_{2}$ is the angle that the first transmitted ray makes with the perpendicular to the normal, $\frac{n_{a} P}{c}$ is the momentum per second transported by light of power $P, R_{e}$ is the fraction of the light intensity reflected from the surface, and $T$ is the fraction of the light intensity transmitted through the surface. For light polarized vertical to the plane of incidence, $R_{e}$ and $T$ are given by the equations:

$$
\begin{gathered}
R_{e p}=\left(\frac{n_{a} \cos \theta_{1}-n_{b} \cos \theta_{2}}{n_{a} \cos \theta_{1}+n_{b} \cos \theta_{2}}\right)^{2} \\
T_{p}=1-R_{e p}
\end{gathered}
$$

For polarization parallel to the plane of incidence, $R_{e}$ and $T$ are:

$$
\begin{gathered}
R_{e s}=\left(\frac{n_{a} \cos \theta_{1}-n_{b} \cos \theta_{2}}{n_{a} \cos \theta_{1}+n_{b} \cos \theta_{2}}\right)^{2} \\
T_{s}=1-R_{e s}
\end{gathered}
$$

where $n_{a}$ and $n_{b}$ are the indices of refraction.

\subsection{Fundamentals of Light Propagation}

Traditionally, optics is divided into four main areas: ray optics, wave optics, electromagnetic optics and quantum optics. The theory of quantum optics can explain nearly all optical phenomena. Electromagnetic optics gives us the most complete treatment of light within the confines of classical optics. Wave optics is a scalar approximation of electromagnetic optics. The wave optics model deals with the wave nature of light itself 
and constitutes a basis for describing some optical phenomena such as interference and diffraction. Ray optics is the limit of wave optics when the wavelength is very short. Ray optics models explain how light travels, and involve the concepts of light reflection, refraction and transmission.

Ray optics is the simplest theory of light propagation, and describes rays that travel in different media and comply with a set of geometrical rules. Rays can be defined by their location and propagation angle. Consequently, this discipline is useful in studying image formation, namely the collection of rays from each point of an object and their redirection by an optical component onto a corresponding point of an image. Optical components are often centered about an optical axis, around which the rays may be treated as traveling at small inclinations. Such rays are called paraxial rays. The change in the position and inclination of a paraxial ray as it travels through a centered optical system can be described by the use of matrix optics. In this thesis, the ray optics model and matrices are used to describe the imaging system of the laser tweezer.

Wave optics is the theory in which light propagation is described by a scalar wave function. Any function satisfying the wave equation represents a possible optical wave. At the boundary between different media, the wave function changes in a way that depends on the refractive index $\mathbf{n}$. For a monochromatic wave, amplitude and phase are generally position dependent, but the wave function is a harmonic function of time with frequency at all positions. There are three wavefront profiles present at specific regions along the beam: planar, spherical and paraxial wavefronts. Planar wavefronts are normal to the optical axis at all points along their surface. Spherical wavefronts propagate radially from a source 
point and diverge in all directions. Paraxial wavefronts can be found along the optic axis of the beam in every region of the beam. Paraxial waves are less divergent than are spherical waves.

The light from a laser can be treated as a kind of Gaussian beam under ideal conditions. The Gaussian beam can be defined such that the beam power is mainly concentrated within a small cylinder surrounding the beam axis while the intensity is distributed in a circularly symmetric Gaussian function centering around the beam axis. The Gaussian beam as a wave also satisfies the wave equation. Gaussian beams can be collimated and focused when transmitted through various optical components such as a lens. In this thesis, the wave optics model and Gaussian beam propagation are used to explain the principle of the laser tweezers.

\subsection{Matrix Optics}

Matrices can be used to model an optical imaging system. For the matrix technique to work the rays must be paraxial, traveling along the beam axis with only small deviation angles. This allows the rays to be described by their vertical positions and angles to the central axis. The matrix takes the ray position and angle when entering the system, applies the component matrices and provides the position and angle of the ray as it exits the system. A simple optical imaging system is illustrated in Figure 3.3 below: 


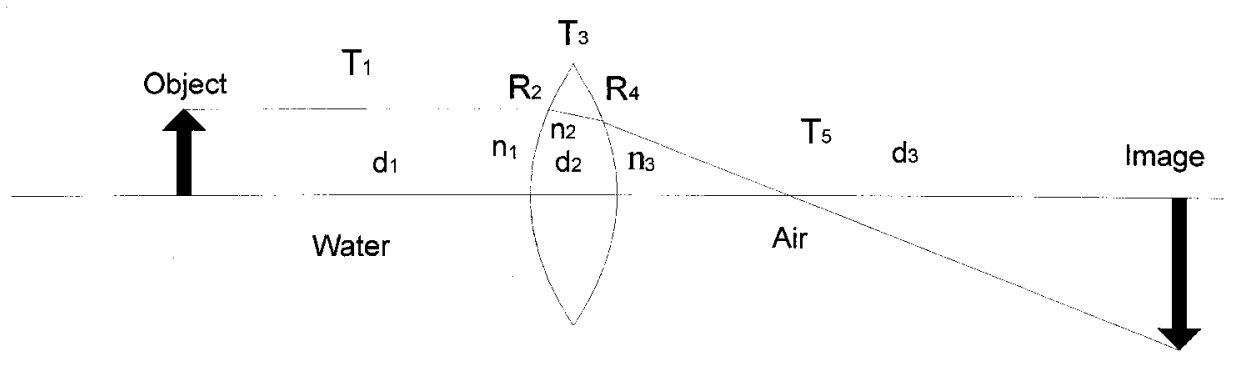

Figure 3.3 Diagram of a simple optical imaging system.

In Figure 3.3, $T_{1}$ is the translation matrix as the light travels $d_{1}$ from the polystyrene sphere to reach the objective lens. $T_{3}$ is the translation matrix as the light travels distance $d_{2}$ in the lens. Finally, $T_{5}$ is the translation matrix as the light travels distance $d_{3}$ from the lens to the CCD camera. $R_{2}$ and $R_{4}$ are the two spherical refractive matrices for the left and right surfaces of the spherical lens.

There are two basic matrices necessary to model an optical imaging system:

a. Translation matrix: $\quad T(d)=\left(\begin{array}{ll}1 & d \\ 0 & 1\end{array}\right)$ where $d$ is the distance the ray is traveling.

b. Spherical refraction matrix: $R(r)=\left(\begin{array}{cc}1 & 0 \\ -\left(\frac{n_{2}-n_{1}}{n_{2}}\right) \frac{1}{r} & \frac{n_{1}}{n_{2}}\end{array}\right)$ where $r$ is the radius of the lens, and $n_{1}$ and $n_{2}$ are the indices of refraction on the left and right side respectively of the lens.

The optical system for this project consists of a separation between polystyrene particles in the simple chamber and the CCD camera. The light enters the system from the 
polystyrene particles to travel distance $d_{1}$ to reach the objective lens. To begin the analysis, a translation matrix can be expressed by:

$$
T_{1}=T\left(d_{1}\right)=\left(\begin{array}{cc}
1 & d_{1} \\
0 & 1
\end{array}\right)
$$

where $d_{1}$ is the distance between the objective lens and polystyrene sphere. The objective lens is assumed to be a thick lens.

The second matrix is a spherical refraction for the first surface of the spherical lens, which is:

$$
R_{2}=\left(\begin{array}{cc}
1 & 0 \\
-\left(\frac{n_{2}-n_{1}}{n_{2}}\right) \frac{1}{r_{1}} & \frac{n_{1}}{n_{2}}
\end{array}\right)
$$

where $r_{1}$ is the curvature of the lens at left side, and $n_{1}=1.33$ and $n_{2}=1.52$ are the indices of refraction of water and glass.

The third matrix is a translation matrix; the light travels distance $d_{2}$ in the lens:

$$
T_{3}=T\left(d_{2}\right)=\left(\begin{array}{ll}
1 & d_{2} \\
0 & 1
\end{array}\right)
$$

The fourth matrix is a spherical refraction for the second surface of the objective lens:

$$
R_{4}=\left(\begin{array}{cc}
1 & 0 \\
-\frac{\left(n_{3}-n_{2}\right)}{n_{3} r_{2}} & \frac{n_{2}}{n_{3}}
\end{array}\right)
$$

where $r_{2}$ is the curvature of the lens at right side, $r_{2}<0, n_{2}=1.52$ is the index of refraction of glass, and $n_{3}=1.00$ is the index for air. 
The fifth matrix is a translation matrix as the light travels distance $d_{3}$ between the objective lens and image on the CCD camera:

$$
T_{5}=T\left(d_{3}\right)=\left(\begin{array}{cc}
1 & d_{3} \\
0 & 1
\end{array}\right)
$$

Therefore, the total optical system matrix can be computed by

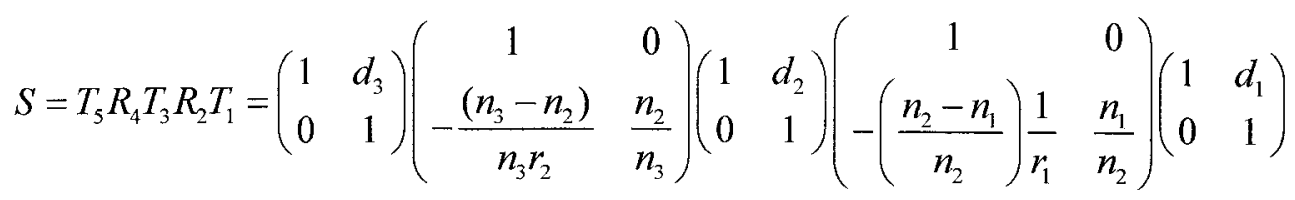

After matrix multiplication, the four elements of the $2 \times 2$ matrix can be written as:

$$
S=\left(\begin{array}{ll}
A & B \\
C & D
\end{array}\right)
$$

where

$$
\begin{aligned}
& A=1+d_{3}-\left(\frac{n_{2}-n_{1}}{n_{2}}\right) \frac{1}{r_{1}}\left(1-\frac{\left(n_{3}-n_{2}\right)}{n_{3} r_{2}} d_{3}\right) d_{2}-\frac{n_{2}}{n_{3}}\left(\frac{n_{2}-n_{1}}{n_{2}}\right) \frac{1}{r_{1}} d_{3} \\
& B=d_{1}\left(1+d_{3}\right)+\left[\left(1-\frac{\left(n_{3}-n_{2}\right)}{n_{3} r_{2}} d_{3}\right) d_{2}+\frac{n_{2}}{n_{3}} d_{3}\right]\left[-\left(\frac{n_{2}-n_{1}}{n_{2}}\right) \frac{1}{r_{1}} d_{1}+\frac{n_{1}}{n_{2}}\right] \\
& C=-\frac{\left(n_{3}-n_{2}\right)}{n_{3} r_{2}}-\left(\frac{n_{2}-n_{1}}{n_{2}}\right) \frac{1}{r_{1}}\left[-\frac{\left(n_{3}-n_{2}\right)}{n_{3} r_{2}} d_{2}+\frac{n_{2}}{n_{3}}\right] \\
& D=-\frac{\left(n_{3}-n_{2}\right)}{n_{3} r_{2}} d_{1}+\left[-\left(\frac{n_{2}-n_{1}}{n_{2}}\right) \frac{1}{r_{1}} d_{1}+\frac{n_{1}}{n_{2}}\right]\left[-\frac{\left(n_{3}-n_{2}\right)}{n_{3} r_{2}} d_{2}+\frac{n_{2}}{n_{3}}\right]
\end{aligned}
$$


By changing the variables $d_{1}, d_{2}, d_{3}, r_{1}$, and $r_{2}$, the optical imaging system can be modeled for different combinations of separation and lens size. Since $r_{1}$ and $r_{2}$ are unknown, we can use a thin lens approximation to convert Figure 3.3 to Figure 3.4.

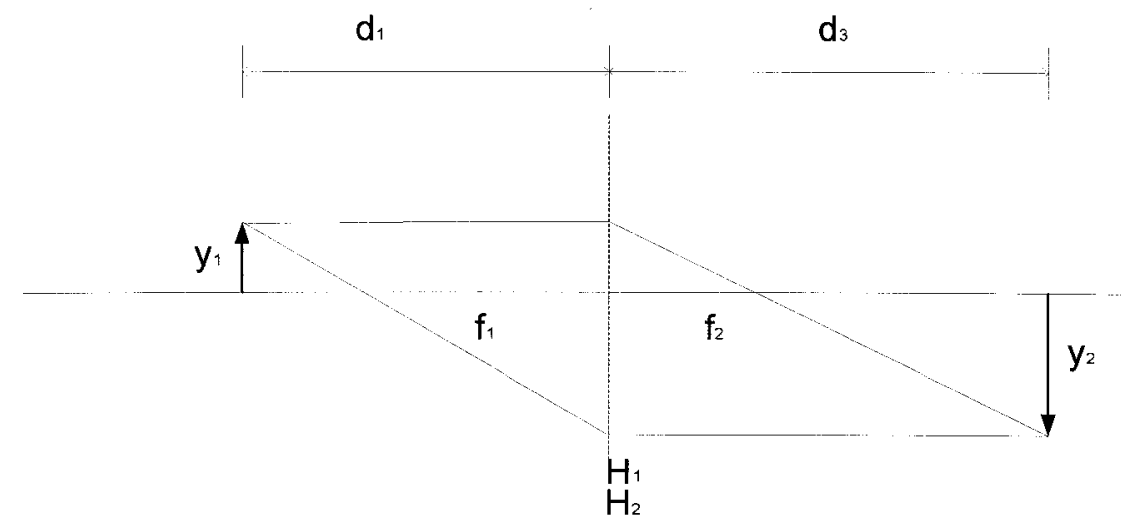

Figure 3.4 Diagram of a simple optical imaging system using thin lens.

where $H_{1}, H_{2}$ are the principle planes and $f_{1}$ and $f_{2}$ are the focal lengths. Using similar triangles we can derive the following two equations:

$$
\begin{aligned}
& \frac{y_{1}}{y_{2}}=-\frac{d_{1}}{d_{3}} \\
& \frac{n_{1}}{d_{1}}+\frac{1}{d_{3}}=\frac{n_{1}}{f_{1}}
\end{aligned}
$$

For typical objective lenses, the effective focal lenses are the following: 
Table 3.1 Effective Focal Lengths for Typical Lenses

\begin{tabular}{|l|c|}
\hline lens & $f_{1}(\mathrm{~mm})$ \\
\hline $10 \mathrm{X}$ & $\mathbf{1 8}$ \\
\hline $20 \mathrm{X}$ & 9 \\
\hline $40 \mathrm{X}$ & 4.5 \\
\hline
\end{tabular}

For CCD camera sensor size $4.8 \mathrm{~mm} \times 3.6 \mathrm{~mm}$, let $y_{2}=4.8 \mathrm{~mm}, y_{2}=3.6 \mathrm{~mm}$ and $d_{3}=500 \mathrm{~mm}$. Combining Eq. (3.8) and Eq.(3.9), we obtain the actual size of the optical image:

Table 3.2 Actual Image Sizes for Typical Objective Lenses

\begin{tabular}{|l|l|l|}
\hline lens & $y_{1}$ (Horiz. $)(\mu m)$ & $y_{1}($ Vert. $)(\mu m)$ \\
\hline $10 \mathrm{X}$ & 178.0 & 133.0 \\
\hline $20 \mathrm{X}$ & 88.0 & 66.0 \\
\hline $40 \mathrm{X}$ & 43.0 & 33.0 \\
\hline
\end{tabular}

The optical image system can be modeled with an objective lens which produces the distances to the polystyrene and CCD camera, the polystyrene size, and the solution medium.

An imaging charge coupled device (CCD) camera connected with BNC cables to a monitor-VCR system is used to observe and record trapping events. To look at the microspheres in the plane of the trap, we need to image onto the spot a small distance from the objective lens. The CCD camera is placed about $500 \mathrm{~cm}$ behind the objective lens. This is illustrated in Figure 3.5, the ray-tracing diagram of the imaging trapped particle. 


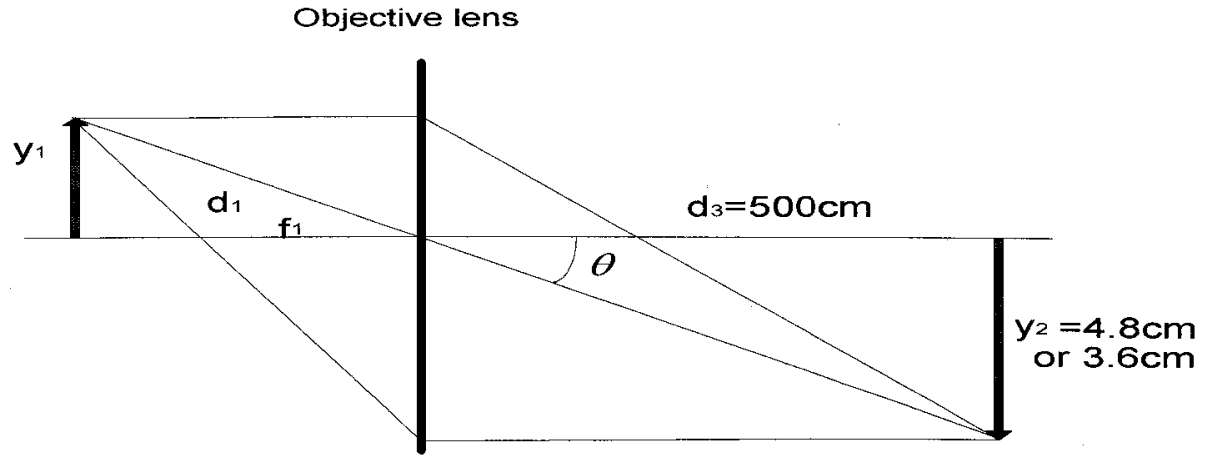

Figure 3.5 Ray-tracing diagram of imaging trapped particle.

\subsection{Gaussian Beam Model}

In most laser applications it is necessary to focus, modify, or shape the laser beam using lenses and other optical elements. Laser-beam propagation can, in general, be approximated by assuming that the laser beam has an ideal Gaussian intensity profile. In order to explain the laser tweezers, it is important to understand the basic properties of Gaussian beam propagation and focusing.

To begin the analysis, we introduce the complex amplitude of a paraxial wave as [21]:

$$
U(r)=A(r) \exp (-j k z)
$$

where $A(r)$ is a complex envelope. The complex envelope of the Gaussian beam is expressed as:

$$
A(r)=\frac{A_{1}}{q(z)} \exp \left[-j k \frac{\rho^{2}}{2 q(z)}\right]
$$


where $z$ represents the axial distance, $\rho=\left(x^{2}+y^{2}\right)^{1 / 2}$ is the radial distance, $k=2 \pi / \lambda$ is the wavenumber, $\lambda$ is the wavelength. $q(z)=z+j z_{0}$, and the parameter $z_{0}$, known as the Rayleigh range, is given by:

$$
z_{0}=\pi W_{0}^{2} / \lambda
$$

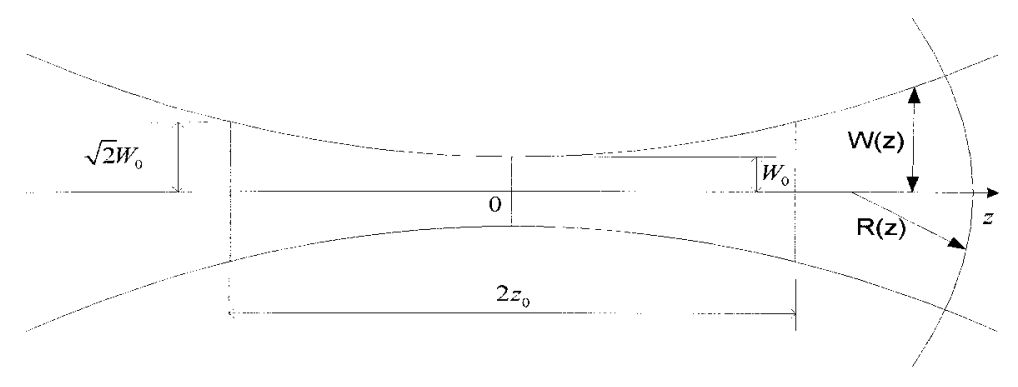

Figure 3.6 The Rayleigh range and depth of focus of a Gaussian beam.

The depth of focus is the axial distance within which the beam radius lies within a factor $\sqrt{2}$ of its minimum value $W_{0}$, called the beam waist radius. The waist diameter $2 W_{0}$ is called the spot size. These can be shown in Figure 3.6. The depth of focus is twice the Rayleigh range $z_{0}$.

$$
\text { Depth of focus }=2 z_{0}
$$

In order to separate the amplitude and phase of this complex envelope, $1 / q(z)$ can be defined using two real functions $R(z)$ and $W(z)$ as:

$$
\frac{1}{q(z)}=\frac{1}{R(z)}-j \frac{\lambda}{\pi W^{2}(z)}
$$

$W(z)$ is a measure of the beam width and $R(z)$ is the wavefront radius of curvature. They are defined as follows: 


$$
\begin{gathered}
W(z)=W_{0}\left[1+\left(\frac{z}{z_{0}}\right)^{2}\right]^{1 / 2} \\
R(z)=z\left[1+\left(\frac{z_{0}}{z}\right)^{2}\right]
\end{gathered}
$$

The beam radius changes along the optic axis. The spot size of the beam at any point along the axis is $2 W(z)$. The radius of curvature, $R(z)$ defines the shape of the wavefront. These are shown in Figure 3.5. Putting equation (3.14) into equation (3.13) and using equation (3.11), the complex amplitude $U(r)$ of the Gaussian beam can be expressed as:

$$
U(r)=A_{0} \frac{W_{0}}{W(z)} \exp \left[-\frac{\rho^{2}}{W^{2}(z)}\right] \exp \left[-j k z-j k \frac{\rho^{2}}{2 R(z)}+j \zeta(z)\right]
$$

and the beam parameters expressed as:

$$
\begin{gathered}
\zeta(z)=\tan ^{-1} \frac{z}{z_{0}} \\
W_{0}=\left(\frac{\lambda z_{0}}{\pi}\right)^{1 / 2}
\end{gathered}
$$

The optical intensity is expressed by substituting equation (3.17) into $I(r)=|U(r)|^{2}$ :

$$
I(\rho, z)=I_{0}\left[\frac{W_{0}}{W(z)}\right]^{2} \exp \left[-\frac{2 \rho^{2}}{W^{2}(z)}\right]
$$

where $I_{0}=\left|A_{0}\right|^{2}$. From equation (3.20), we can see that the intensity is a Gaussian function of the radial distance $\rho$ at each axial distance $z$. 
If the Gaussian beam width $W(z)$ and the radius of curvature $R(z)$ are known at some point on the beam axis, we can determine the location $z$ and beam waist radius $W_{0}$ by combining equation (3.15) and equation (3.16):

$$
\begin{gathered}
z=\frac{R}{1+\left(\lambda R / \pi W^{2}\right)^{2}} \\
W_{0}=\frac{W}{\left[1+\left(\pi W^{2} / \lambda R\right)^{2}\right]^{1 / 2}}
\end{gathered}
$$

When a diverging beam is incident on a positive lens, it will be focused to a new waist on the opposite side. Figure 3.7 shows a Gaussian beam centered at $z=0$ with waist radius $W_{0}$ transmitted through a thin lens located at distance $z$ to get another waist radius $W_{0}^{\prime}$.

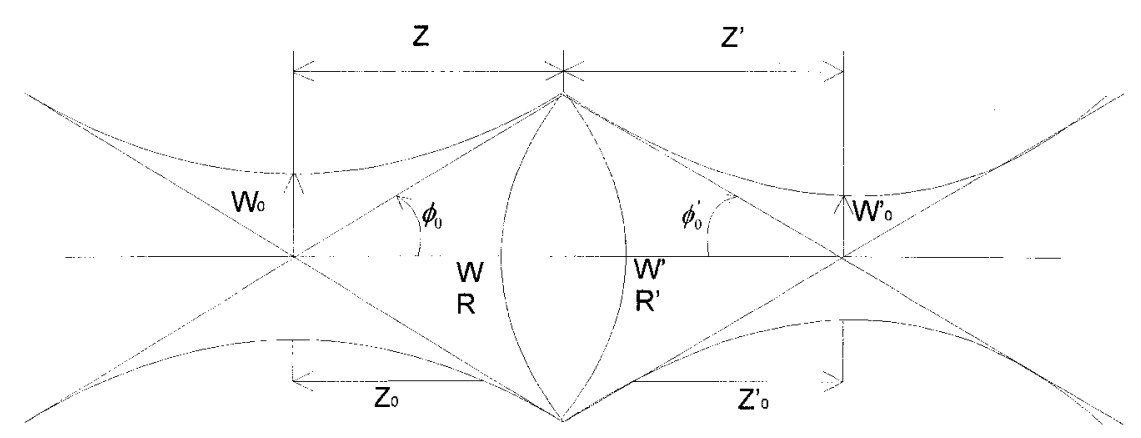

Figure 3.7 Focusing a Gaussian beam through a lens.

By use of equation (3.21) and equation (3.22), the new beam waist radius is:

$$
W_{0}^{\prime}=\frac{W}{\left[1+\left(\pi W^{2} / \lambda R^{\prime}\right)^{2}\right]^{1 / 2}}
$$

and the beam waist centers at the location: 


$$
-z^{\prime}=\frac{R^{\prime}}{1+\left(\lambda R^{\prime} / \pi W^{2}\right)^{2}}
$$

For a thin lens of focal length $f$, there is:

$$
\frac{1}{R}=\frac{1}{R}-\frac{1}{f}
$$

Substituting equation (3.15) and equation (3.16) into equation (3.23) to equation (3.25), the relationship between the beam parameters before and after the lens are as follows:

Waist radius:

$$
W_{0}^{\prime}=M \cdot W_{0}
$$

Waist location:

$$
z^{\prime}-f=M^{2} \cdot(z-f)
$$

Depth of Focus:

$$
2 z_{0}{ }^{\prime}=M^{2} \cdot\left(2 z_{0}\right)
$$

Divergence:

$$
2 \theta_{0}^{\prime}=\left(2 \theta_{0}\right) / M
$$

Magnification:

$$
M=M_{r} / \sqrt{1+r^{2}}, \quad r=\frac{z_{0}}{z-f}
$$

where $r=z_{0} /(z-f)$ is the radius at $z, W_{0}$ is the radius of beam waist before the lens and $W_{0}^{\prime}$ is the radius of beam waist after the lens. $M_{r}=|f /(z-f)|$ is the transverse magnification. 
A primary use of lenses is to focus down a beam to a much smaller spot size. This can be obtained by locating the lens at the minimum waist of the beam, causing it to be focused to a smaller minimum waist. See Figure 3.8 below:

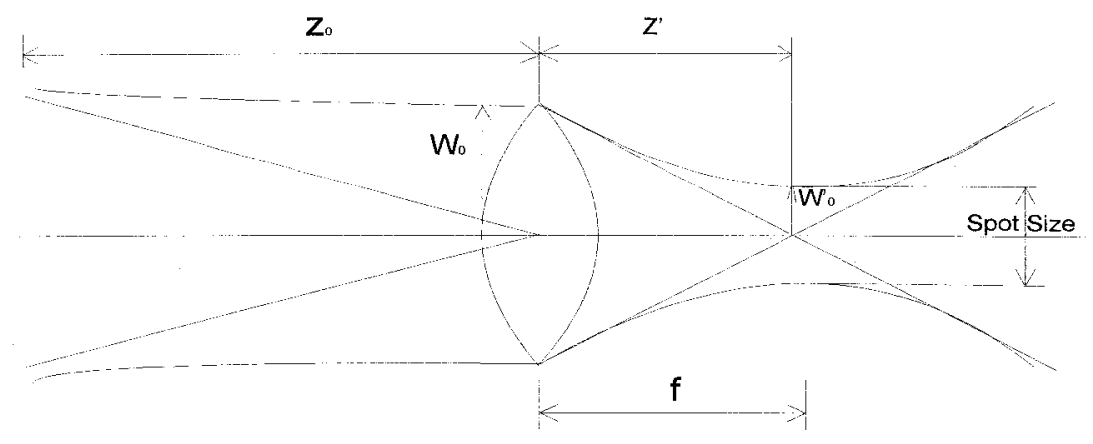

Figure 3.8 Focusing a Gaussian beam through a lens.

Substituting $z=0$ into equations (3.26a) through (3.26e), the Gaussian beam transmits through the lens and is focused to a waist radius $W_{0}^{\prime}$ at a distance $z^{\prime}$ given by:

$$
\begin{gathered}
W_{0}^{\prime}=\frac{W_{0}}{\left[1+\left(z_{0} / f\right)^{2}\right]^{1 / 2}} \\
z^{\prime}=\frac{f}{1+\left(f / z_{0}\right)^{2}}
\end{gathered}
$$

When the depth of focus of the incident Gaussian beam $2 z_{0}$ is much longer than the focal length $f$ of the lens, using equation (3.12), equation (3.27) and equation (3.28) we can approximate the following:

$$
W_{0}^{\prime} \approx \frac{\lambda}{\pi W_{0}} f
$$




$$
z^{\prime} \approx f
$$

The small divergence angle of the beam can be approximated by:

$$
\sin \theta \doteq \tan \theta=\frac{W_{0}}{f}
$$

Substituting equation (3.31) into equation (3.29), we obtain:

$$
W_{0}^{\prime}=\frac{\lambda n}{\pi(N A)}
$$

where $N A=n \sin \theta$ is the numerical aperture of the microscope objective, $n$ is the index of refraction of the medium ( $n=1.33$ for water) and $\theta$ is the half-angle of the focused cone of light. Using expression $z_{0}=\pi W_{0}^{2} / \lambda$, the depth of focus is obtained:

$$
z^{\prime} \doteq \frac{\lambda n^{2}}{\pi(N A)^{2}}
$$

The following Table 3.3 gives the beam waist and the focus of depths for three typical objective lenses at $\lambda=785 \mathrm{~nm}$ used in the laser tweezer system.

Table 3.3 The Beam Waist Radius and Depth of Focus for Three Typical Lenses

\begin{tabular}{|l|l|l|l|}
\hline lens & NA & $W_{0}^{\prime}(\mu m)$ & $2 z^{\prime}(\mu m)$ \\
\hline $10 \mathrm{X}$ & 0.25 & 1.32 & 14.16 \\
\hline $20 \mathrm{X}$ & 0.40 & 0.83 & 5.52 \\
\hline $40 \mathrm{X}$ & 0.65 & 0.51 & 2.10 \\
\hline
\end{tabular}

Based on the above analysis of the Gaussian beam and imaging system, the following chapter describes the various optical components used to set up the laser tweezers and the essential techniques involved in the experiments. 


\section{Chapter 4}

\section{Experiment}

\subsection{Top-down Laser Tweezers}

The top-down laser tweezer is one of the standard optical configurations for laser trapping. The laser beam enters the sample chamber from above, travels downwards through the solution and is incident onto the polystyrene micro-sphere on the bottom of the micro-strip glass base of the sample chamber. In this configuration, the polystyrene micro-spheres in suspension can be pushed onto the bottom of the chamber and further manipulated optically.

This chapter discusses the components used in the top-down laser tweezer, the system adjustments and the manipulation of micro-spheres. A brief discussion of template preparation used as the base of the sample chamber is also given. Figure 4.1 shows the schematic of the Top-down laser tweezer experimental setup. Figure 4.2 shows a picture of the Top-down laser tweezers setup and identifies component locations. 


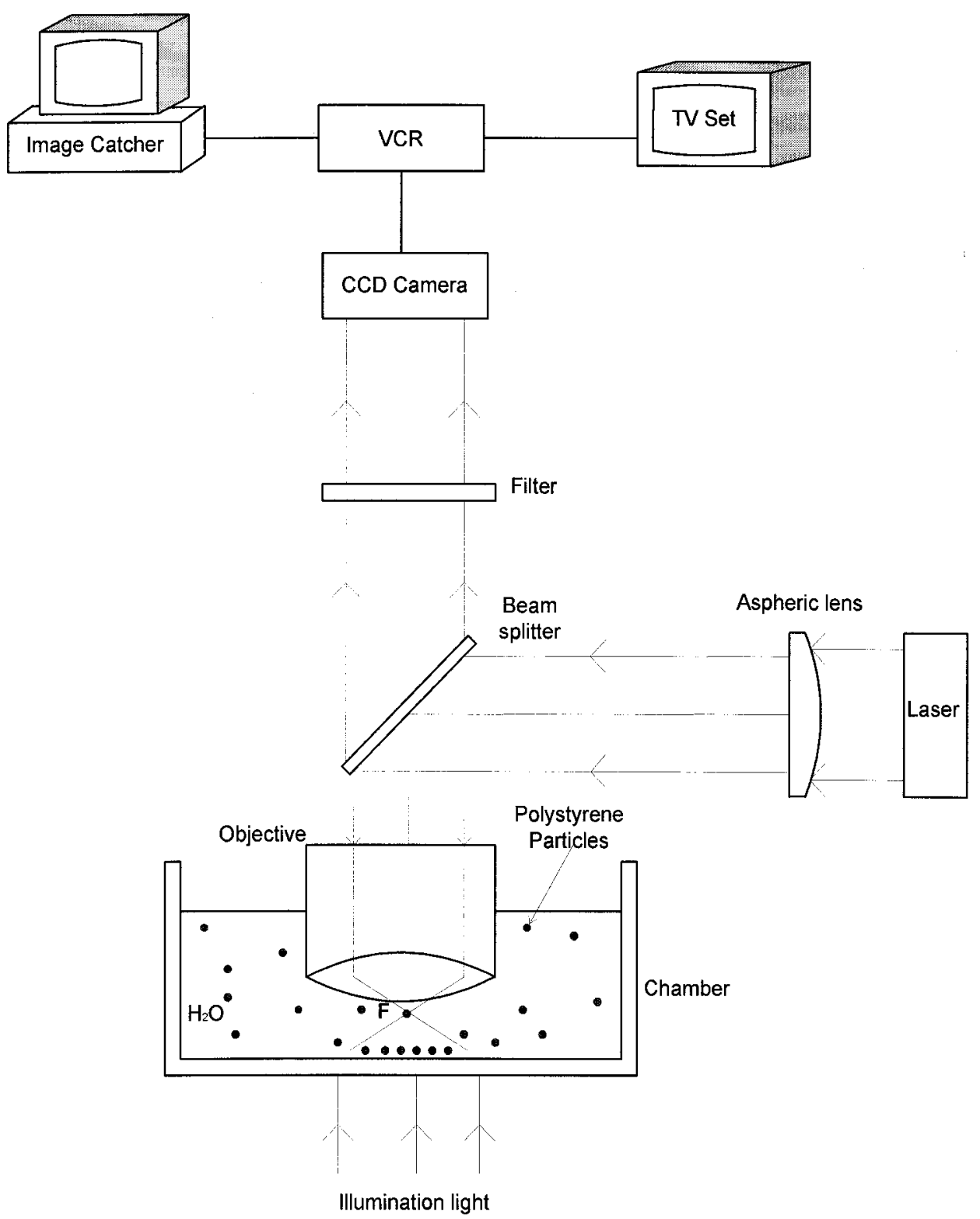

Figure 4.1 Schematic of the top-down laser tweezers experimental setup. 


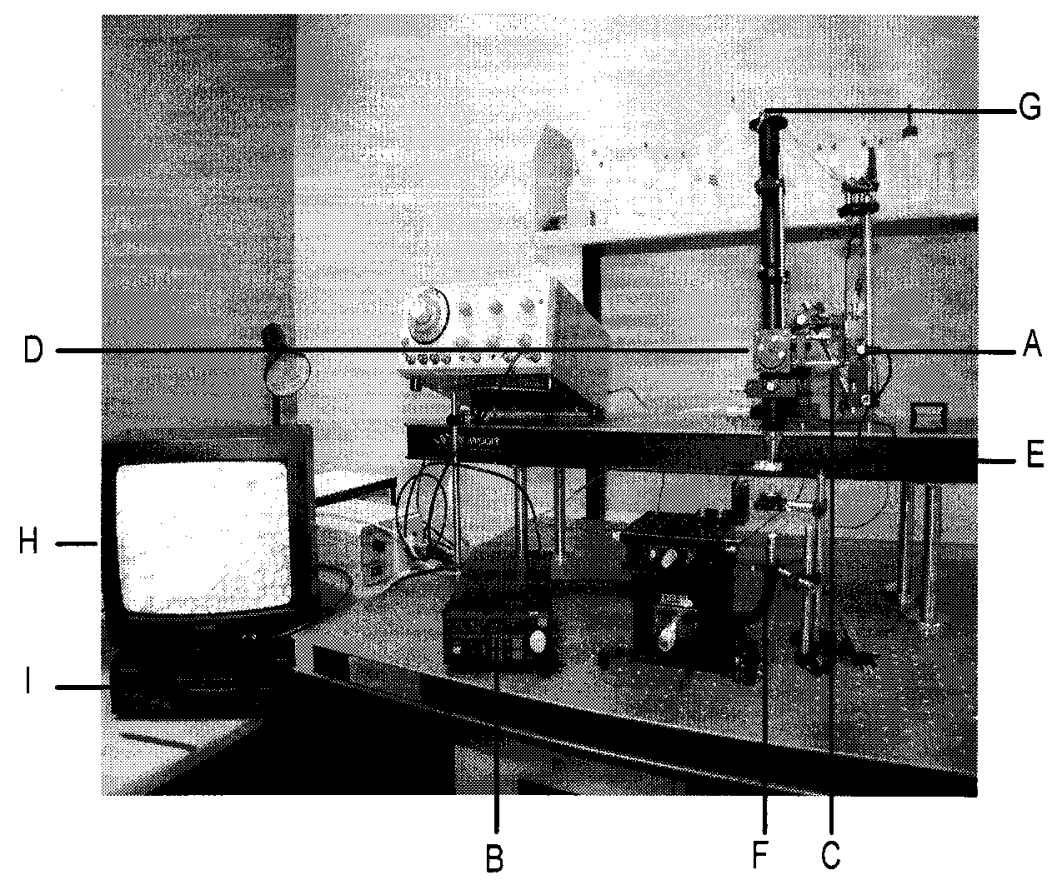

Figure 4.2 Major components shown in the top-down laser tweezers setup.
A: Laser diode
B: Laser diode drivers
C: Collimator lenses
D: Beam splitter
E: Objective
F: Light Source
G: CCD camera
H: TV
I: VCR

\subsection{Equipment Used in Laser Tweezers}

The major components required in a Top-down laser tweezer system are shown in Figure 4.1. The laser tweezer system consists of a laser diode $(\lambda=785 \mathrm{~nm}, P=70 \mathrm{~mW}, I=100 \mathrm{~mA})$, laser illumination, laser diode driver (LCD 500), collimator lenses, beam splitter, and objective. The image capture system consists of a light source, CCD camera, TV and VCR, ATI image catcher, and computer.

Other minor components needed to mount laser tweezers are given in the Appendix. Some major components are shown below: 


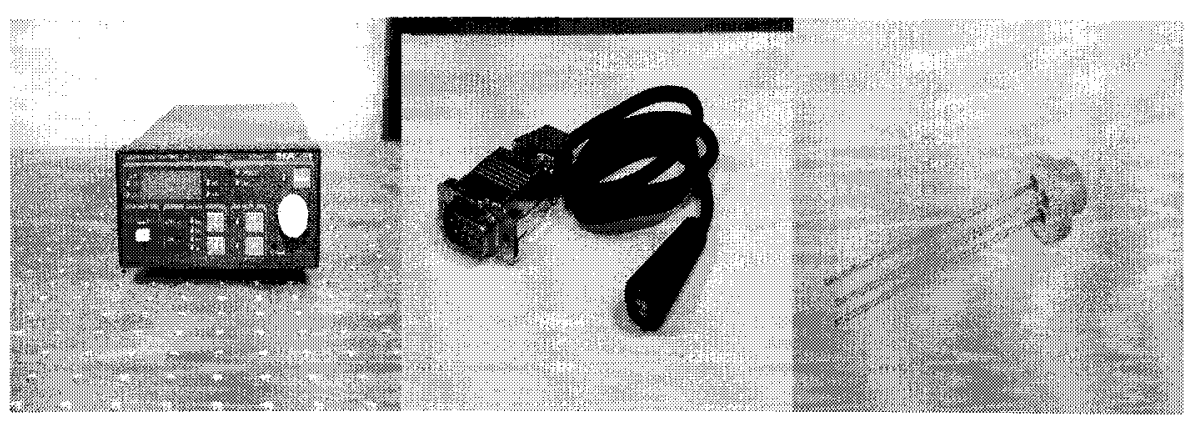

Figure 4.3 Laser diode drivers (LDC 500), the DB9 interface cable and laser diode $(\lambda=785 \mathrm{~nm}, P=70 \mathrm{~mW}, I=100 \mathrm{~mA})$.

The laser diode driver generates a constant current for the laser diode operation. In this experiment, we set the current limit to $100 \mathrm{~mA}$ to meet the limitations and specifications of the laser. A DB9 cable is used to connect the laser diode driver and laser diode.

The laser diode provides a maximum optical power output of $70 \mathrm{~mW}$ at a wavelength of $785 \mathrm{~nm}$. There are three pins, the laser anode, common case and monitor diode cathode, connected to a DB9 interface cable. The laser diode is mounted on the laser diode mount shown below.

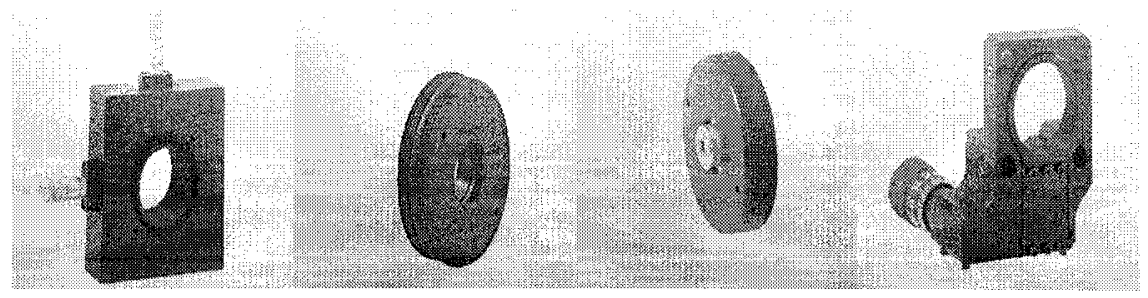

Figure 4.4 XY translator, aspheric lens adaptor, laser diode mount and Z translator. 
The $X Y$ translator is a component used to mount an aspheric lens to collimate the laser beam and hold the laser diode mount. The $\mathrm{XY}$ translator is used to move the laser beam in the XY plane and focus the laser beam as needed.

The $Z$ translator holds a collimating lens in the vicinity of the laser diode. The $1.5 \mathrm{~mm}$ of travel along the axis of the laser beam enables a precise adjustment of the laser beam focus in the sample region.

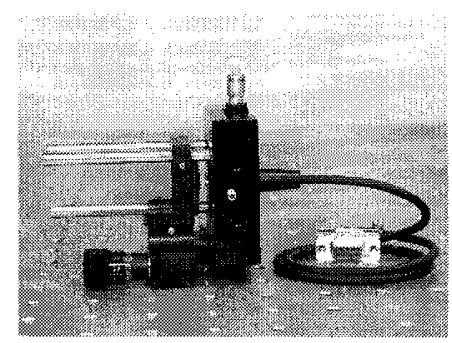

Figure 4.5 Laser beam collimation setup

Figure 4.5 shows a picture of the laser beam collimation setup using the components of Figure 4.4, the laser diode and the aspheric lens (shown is Figure 4.7). Collimation is the process of converting the diverging output of the laser beam from the laser diode into a beam of parallel light. An aspheric lens ( $f=4.5 \mathrm{~mm}, 0.55 \mathrm{NA})$ designed for the wavelength $780 \mathrm{~nm}$ is used. This aspheric lens was placed into the $Z$ translator and then placed in front of the laser diode holding on the XY translator. In order to get a parallel beam, the end-face of the laser should be placed at the focal plane of the aspheric lens as shown by the diagram in Figure 3.8. The aspheric lens is shown in Figure 4.7. Figure 4.6 demonstrates the collimation process with a laser diode. 


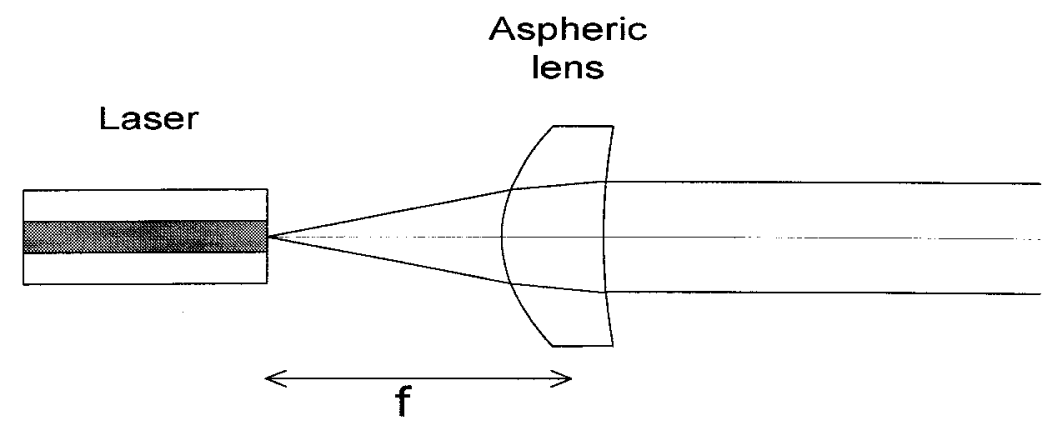

Figure 4.6 Collimation of divergent beam.
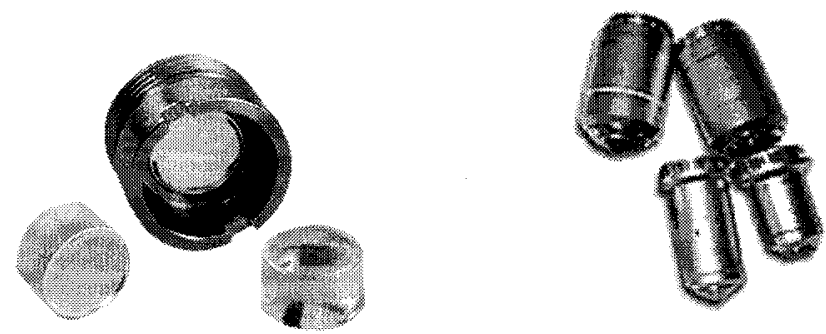

Figure 4.7 Aspheric lens and objective lens.

The objective lens is a key component to forming the laser trap. It has two main functions. The first function is to focus the incoming laser beam to generate a trapping environment. The second function is to magnify the sample to generate clear images. Three types of immerse objective lenses (10X, 20X, and 40X) were used. The objective lens is shown in Figure 4.7. 

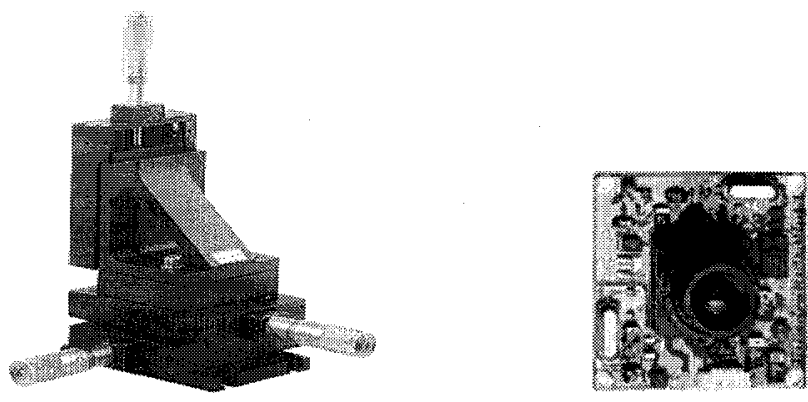

Figure 4.8 XYZ translation stage and CCD camera.

The XYZ translation stage ensures perpendicularity among the three axes. It provides stability and precision, and is capable of sustaining heavy loads. The laser tweezers is mounted on the XYZ translation stage. Through the three axes of motion, the laser tweezers can be adjusted within the sample chamber to create the trapping environment. A CCD camera was used to take the image of the trapping process. It uses a small, rectangular piece of silicon called a charge-coupled-device (CCD). The sensing area is $4.8 \times 3.6 \mathrm{~mm}$. These are shown in Figure 4.8.
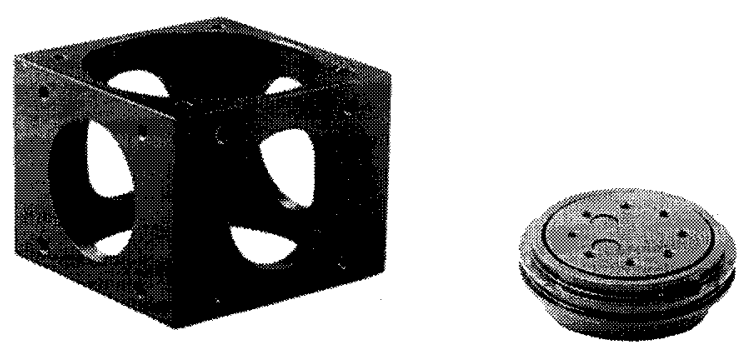

Figure 4.9 Cage cube and kinematics mounting platform. 
The cage cube is another important component in the laser tweezers setup. It is used in conjunction with extension rods, the kinematics mounting platform, objective lens, collimated laser and CCD camera. The kinematics platform is used to install the beamsplitter. These two components are shown in Figure 4.9.

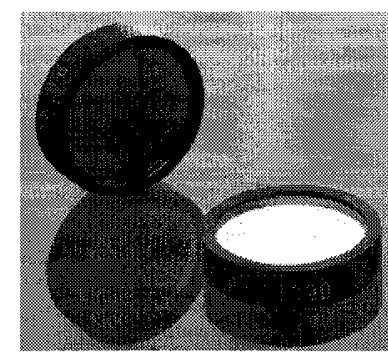

Figure 4.10 Beam splitter and filter.

The beamsplittter (35-5346-CRR) is used to produce a 50:50 splitting ratio for the laser beam. It was mounted on the kinematics mounting platform. The filter (35-5388TCRR) is used to block strong laser beams from entering the CCD camera. These components are shown in Figure 4.10.
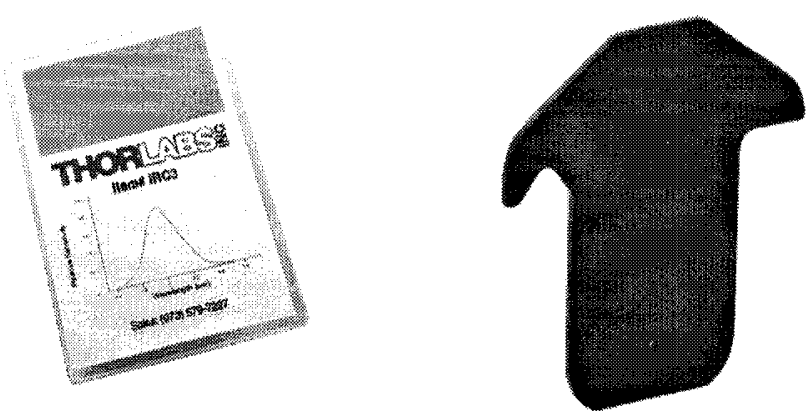

Figure 4.11 IR viewing card and alignment tool for cage system. 
The IR viewing card provides an instant visible pattern for determining beam location and beam size. The card uses phosphors that must be charged in room light in order to see the pattern. The alignment tool is used to center the laser beam on the main axis within the assembly. These are shown in Figure 4.11.

\subsection{The Laser Beam Delivery System Setup}

As noted in Section 3.4, the Gaussian beam must be focused to a small spot size. For three typical objective lenses (laser wavelength $\lambda=785 \mathrm{~nm}$ ), the spot sizes of the focused laser beam are $2.64 \mu m$ (10X objective lens), $1.66 \mu m$ (20X objective lens) and $1.02 \mu m$ (40X objective lens).

In Figure 4.1, we can see that the laser beam emits from the laser diode, and since the laser beam is diverging, the aspheric lens is used to collimate the laser beam into a parallel light beam. After using a beam splitter to direct the parallel light beam down to an objective lens, the laser beam can be highly focused to a small waist beam reaching the bottom of the sample chamber. Having already examined the installation of the imaging system, the detailed setup of the laser beam delivery system is given below.

The laser diode is connected to the laser driver (LDC500) by the DB9 interface cable. One end of the DB9 cable contains a three-pin diode holder held in an XY translator. The translator, in turn, is fixed to the end of the cage assembly rods. An aspheric lens ( $\mathrm{f}=4.5 \mathrm{~mm} 0.55 \mathrm{NA}$ ) is mounted on the $\mathrm{Z}$ translator, held in the cage assembly rods after the XY translator. The spacing between the aspheric lens and endface of the laser diode was less than $4.5 \mathrm{~mm}$. As mentioned above, the cage cube has 
been connected to the objective lens and CCD camera. The laser beam is aligned by adjusting the $\mathrm{XY}$ translator and the $\mathrm{Z}$ translator to penetrate the small center hole of the alignment tool for cage assembly. An IR viewing card is then used to determine the beam location and approximate intensity spot size. The beam waist location of the intensity laser spot can be obtained by moving the $Z$ translator. The same cage assembly rods holding the $\mathrm{XY}$ translator and $\mathrm{Z}$ translator are fixed to the right side of the cage cube. A kinematics mounting platform holding the beam splitter (35-5346-CRR) is fixed at $45^{\circ}$ at the center of the cage cube by the prism mounting platform. This kinematics mounting platform with the beam splitter is fixed on the front side of the cage cube. The $\mathrm{XY}$ translator above the objective is used to adjust the parallel beam to transmit through the center of the objective lens. These are shown in Figure 4.12.

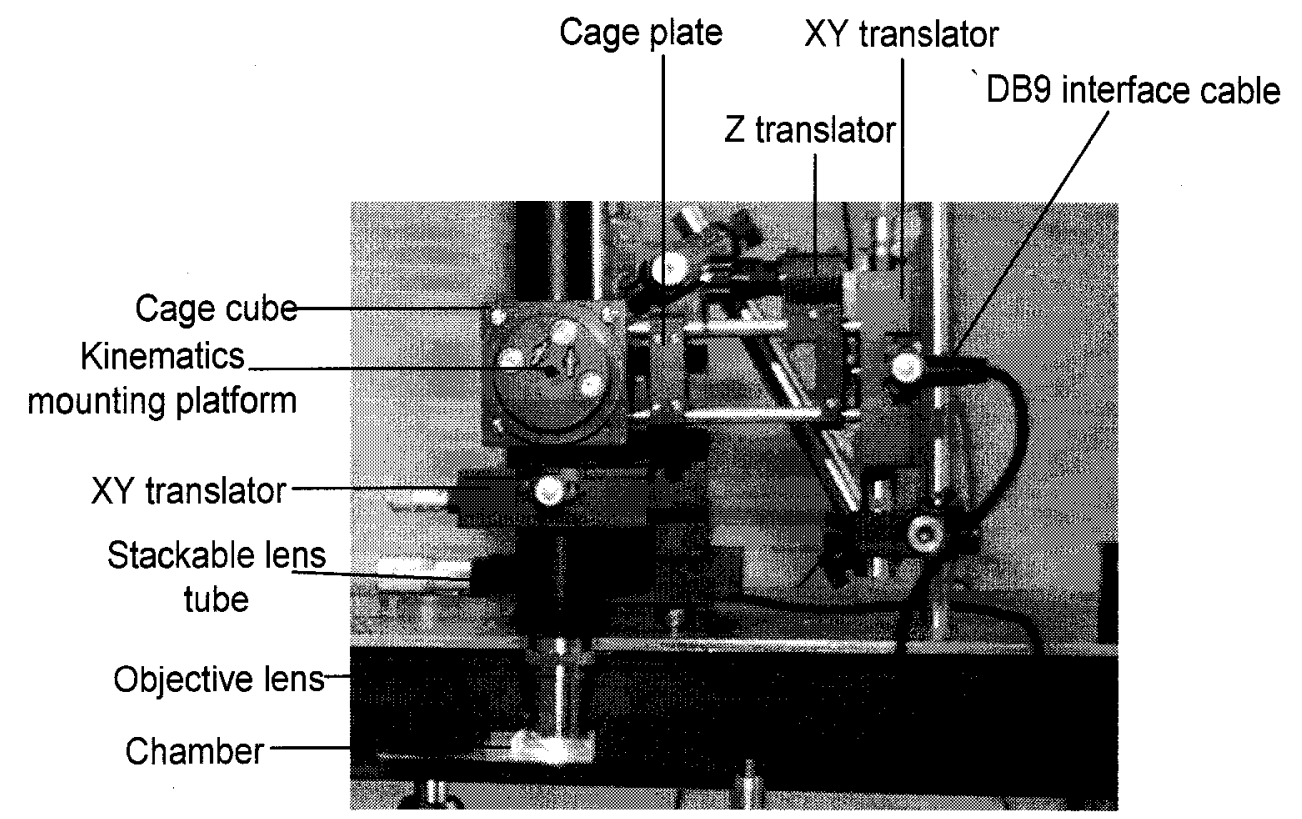

Figure 4.12 Laser beam delivery system of the top-down laser tweezers setup. 


\subsection{Imaging System Setup}

As described in Section 3.3, once the CCD camera and objective lens are selected, the actual viewing image size depends on the fixed distance between the CCD camera and objective lens. For a CCD camera sensor size of $4.8 \mathrm{~mm} \times 3.6 \mathrm{~mm}$, the distance between the CCD camera and the objective lens was set to $510 \mathrm{~mm}$, and the actual image viewing sizes were $166.0 \mathrm{~mm} \times 125.0 \mathrm{~mm}$ (for the $10 \mathrm{X}$ objective lens), $82.0 \mathrm{~mm} \times 62.0 \mathrm{~mm}$ (for the $20 \mathrm{X}$ objective lens), $40.0 \mathrm{~mm} \times 30.0 \mathrm{~mm}$ (for the $40 \mathrm{X}$ objective lens).

In Figure 4.12, image tracing is introduced by following the steps described below. First, the illuminating light from the Maglite is collimated by a collimation lens. The collimated light travels through the glass bottom of the sample chamber and allows the viewing image to be seen clearly through the CCD camera. Second, the viewing image is magnified through an objective lens sitting above, and the chip sensor of the CCD camera picks this up. The CCD camera is connected to a TV-VCR system to observe and record trapping images.

The installation of the imaging system requires three separate components: the illumination component, the chamber component and the combined objective lens and CCD camera component. Setup preparation was done using an optical breadboard. The installation procedure related to Figure 4.13 is now described:

a. The illumination component: The illuminating Maglite was put on another table in order to avoid the vibration caused by the fan inside the illuminator. The illuminating light is delivered by a fiber optic light guide. The fiber optic 
light guide was fixed using a V-Clamp, posts and post holders mounted on an optical breadboard, directing the illuminating light vertically upward. A collimation lens was also fixed using a lens holder, posts and post holders mounted on an optical breadboard. Adjusting the fiber optic light guide and collimation lens increases the intensity of the illuminating light on the sample chamber. These are shown in Figures 4.2 and 4.13.

b. The chamber component: The bottom of the chamber is made of micro-strip glass, while the container walls are made using Mylar transparent plastic. The chamber was clamped using a magnetic kinematic base plate. Then, the magnetic base plate is supported by a post and fixed on an XYZ flexure stage. A level was used to make the base of the chamber in the water-level plane. The chamber can be controlled to move along the $\mathrm{X}, \mathrm{Y}$ and $\mathrm{Z}$-axes. This is shown in Figure 4.13.

c. The combined objective lens and CCD camera component: This component is more complicated, as it uses various mechanical and optical devices. The objective lens was fixed using a stackable lens tube. The other end of the stackable lens tube is connected to an $\mathrm{XY}$ translator, which in turn is connected to the bottom of the cage cube by a lens coupler. The XY translator and cage cube were adjusted so that the front side of the cage cube was in the $\mathrm{Y}$-axis and the left side was in the $\mathrm{X}$-axis of the $\mathrm{XY}$ translator. An extension tube was connected to fix on the top side of the cage cube. In order to be able to capture a clear image of the polystyrene, we had to install one absorptive 
filter (35-5388T-CRR), positioned above the cage cube and below the CCD camera. The purpose of the filter was to block laser light from reaching the CCD camera while still obtaining a clear image of the polystyrene particles.

One cage plate and three rods were used to mount around the extension tube. The filter was mounted on the rod, the position of which is shown in Figure 4.13. Another extension tube was fixed beneath the cage plate. Finally, the CCD camera was mounted on the other end of the third extension tube, mounted on the cage plate. The distance between the CCD camera and objective lens was $510 \mathrm{~mm}$. A CCD camera was connected with BNC cables to a TV-VCR system to observe and record trapping. These are shown in Figures 4.2 and 4.13.

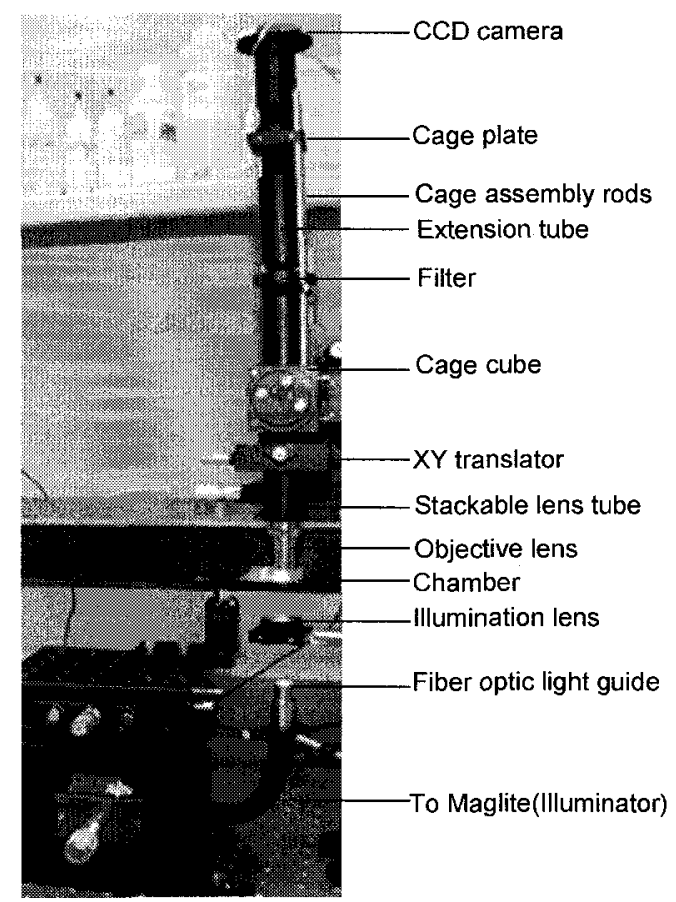

Figure 4.13 Imaging system of the top-down laser tweezers setup. 
Until now, the laser diode, aspheric lens, beam splitter, objective lens and CCD camera have all been mounted together as a unit through the cage cube assembly. We call this unit a laser tweezers. One XYZ translation stage mounted on a medium-sized optical breadboard and supported by rods constitutes another plane on which to mount the Top-down laser tweezers. This makes it easier to manipulate the laser tweezers. The $\mathrm{XYZ}$ translation stage, which is made of steel and is easy to reconfigure with steel linear bearings for $\mathrm{X}, \mathrm{Y}$ and $\mathrm{Z}$-axis motion, is used to hold the laser tweezers. This stage makes it possible to focus the laser beam and CCD camera, and is shown in Figure 4.2.

\subsection{Data Gathering}

Having set up the laser trap experiment set-up was completed, the next challenge was to construct a method for obtaining data for analysis. A monitoring TV was used to view the intensity of the laser beam and laser trap. Sample polystyrene particles and images were recorded on VCR tape for later analysis. The images were then captured from the CCD camera and sent to the monitor-VCR for recording, while we simultaneously observed the picture on the monitoring TV. Since the fabrication of colloidal photonic crystals is a long process, not all the pictures were useful. A PC-driven parallel input video capture card was utilized to capture the data stored in JPG format for screening. An ATI graphics card provided the necessary capabilities to meet our requirements. Figure 4.14 shows the entire data gathering apparatus. 


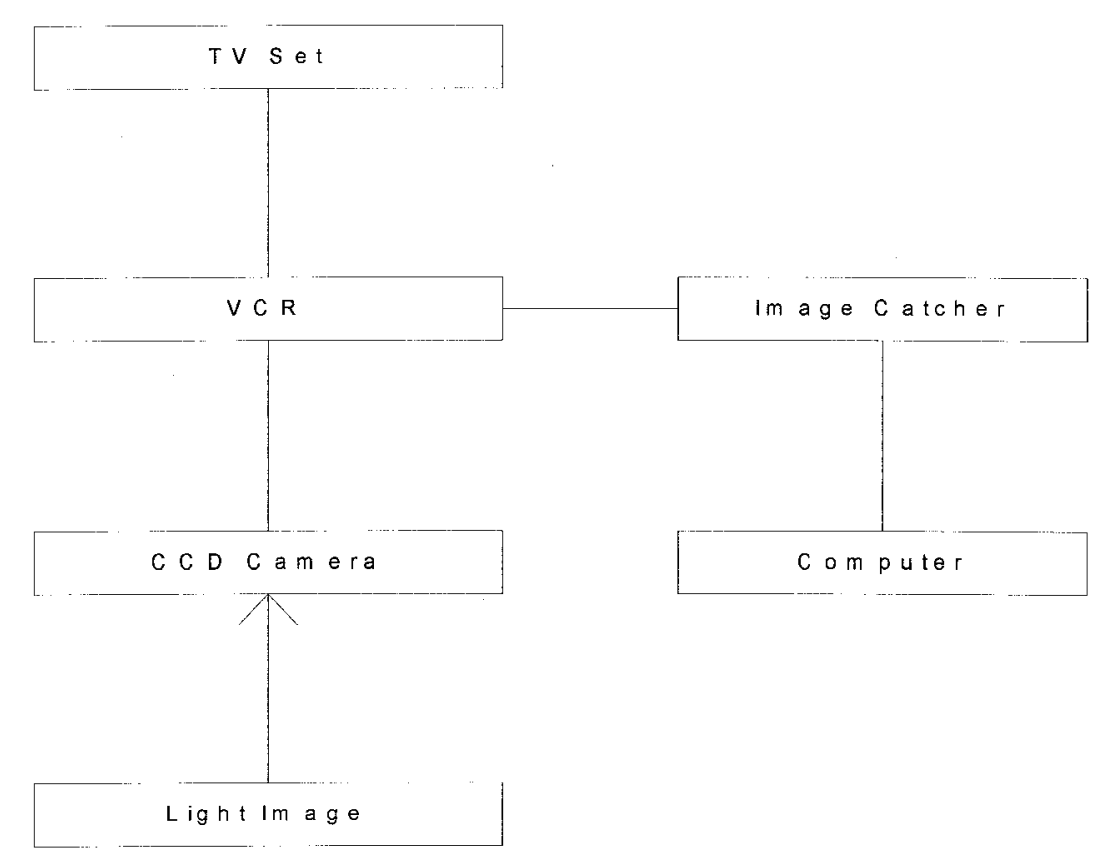

Figure 4.14 Schematic of data gathering.

\subsection{System Adjustments}

In setting up and manipulating the laser trap, there were some adjustments required:

a. Focusing the cameras: To record experimental observations, the camera had to be incorporated into the laser trap design. Initially, dust was observed on the monitor. Lens cleaning paper was used to swipe the surface of the camera. Next, the laser spot was not in the right place on the monitor. Adjusting the angle of the camera positioned the laser spot in the center of monitor picture. Finally, when the laser trap was set up, the monitor screen was so bright that the micro-spheres could not be seen. In order to solve this problem, an absorptive filter was used to prevent the laser light from entering the camera. 
b. Laser focusing: Absent a carefully constructed method, the laser beam proved difficult to manipulate in a way that met the requirements of the experiment. A good laser trap was often difficult to obtain. The laser beam was focused by placing it in an adjustable $\mathrm{X}-\mathrm{Y}$ axis oriented to the center of the reflecting lens. A filter was used in the cube cage to position the beam at an angle of 90 degrees. Adjustments were also made to ensure the laser beam traveled through both pinholes in the alignment plate. The filter was then adjusted by hand to get the laser beam close to the center of the objective lens. We also had to ensure that the laser beam went through the pinhole in the alignment plate in the direction of the objective lens. At the same time, the $\mathrm{X}-\mathrm{Y}$ axis orientation was also adjusted to ensure the laser beam traveled through the center of the objective lens.

c. Laser and camera matching: Although the camera and laser had been set up, a clear picture of laser trap focusing and particles was not easy to obtain. This turned out to be a problem of laser and camera matching. A clear picture of the polystyrene particles had to be formed. By adjusting and moving the camera and adjusting the laser beam, a laser trap could be seen as a small spot on the monitor trapping the particles.

d. Vibration of the system: Since the diameter of the polystyrene micro-sphere particles was in the micrometers range, any vibration from the system components led to the vibration of particles in the chamber, thereby interfering with the fabrication of the photonic crystal structures. The VCR, laser driver and maglite were positioned on another table to avoid the vibrations from cooling fans and other components. 
Another problem was vibrations in the environment outside the system. Conducting the experiment at night was a good way to circumvent this problem.

\subsection{Manipulation of Colloidal Particles in Chamber}

The chamber was an important part of the experiment. Micro-strip glass was chosen as the base glass and Mylar ${ }^{\circledR}$ polyester film as the walls of the chamber. Instant glue was used to tightly bind the glass and polyester. After the laser was lined up, the installation of the polystyrene solution in the chamber for the manipulation of microspheres was another important step. The chamber was placed in a magnetic clamp and situated onto the objective lens, enabling the minimum waist of the laser beam to be moved in the base plane of the micro-strip chamber. The chamber allowed for the displacement of the beam waist and polystyrene sample volume. The chamber also ensured optical alignment such that X10, X20, and X40 objective lenses could be placed in the chamber.

The $2.0 \mu m$ polystyrene latex particles (Polysciences Inc.) were trapped and used for the optical directed epitaxy of colloidal crystals. The particle density had to be optimized in the sample. As a result of their high densities, the particles were difficult to separate. Several particles were easily trapped at the same time. Usually, a drop of concentrated particles equal to $5 \mu \mathrm{l}$ was used. Three drops of concentrated polystyrene particles were added to about $60 \mathrm{ml}$ of water in a test bottle and shaken. Three to five drops of solvent were added into $3 \mathrm{ml}$ of water in the chamber. The chamber was then held firmly in place under the laser tweezers using magnetic clips; the objective lens 
focused on it, and was immersed in the sample solvent in the chamber. Through the illumination provided by an intense power source, the laser tweezers were focused onto the bottom plane containing the particles. The position of the light source was adjusted to provide optimal contrast. The particles were observed on the monitoring TV set via the CCD camera.

\subsection{Preparation of Patterned Template}

Although colloidal crystals can be grown using a non-patterned substrate, patterning substrates is a very important approach for the growing of colloidal crystals. Apart from resorting to UV-light photolithography in the preparation of pattering substrates, a nonmask dual beam multiple exposure technique has also successfully produced templates for the growth of colloidal crystals. The optical system that produces patterns for the growth of colloidal crystal is shown in Figure 4.15. A diffractive grating is used to divide the blue line (488nm) of the Argon Ion laser beam into two beams of equal intensity. Each beam is allowed to expand and is directed onto a three-axis rotation and translation platform where the photoresist sample is mounted. An interference pattern of high and low intensity lines is produced in the photoresist sample. 


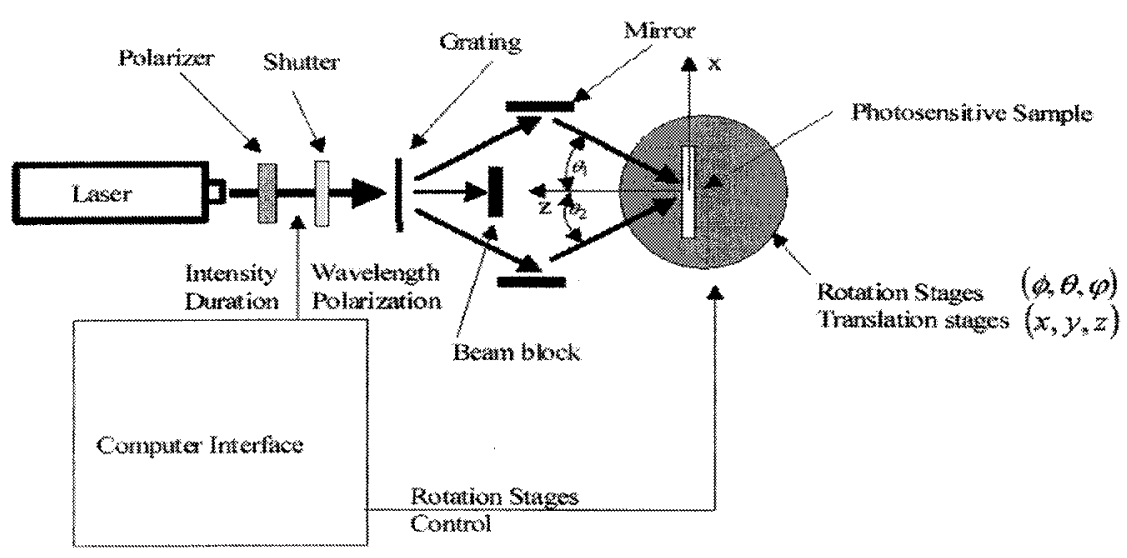

Figure 4.15 Experimental dual beams multiple exposure optical system [12].
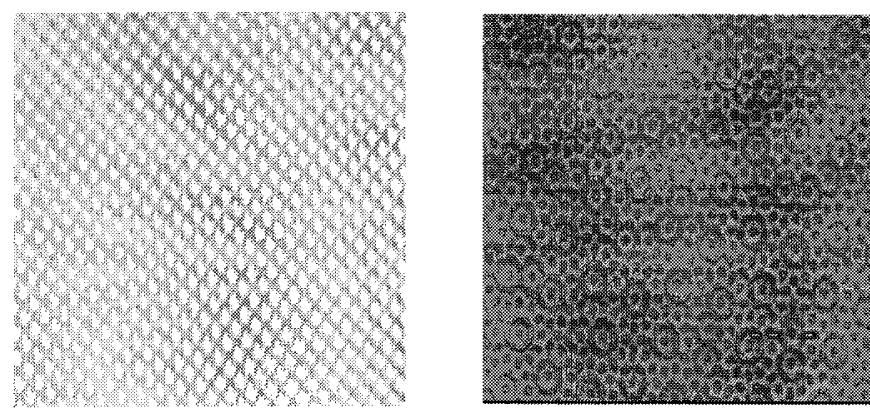

Figure 4.16 Photonic crystal pattern substrate (left) and quasi-crystal pattern substrate (right) [12].

The above figure shows the patterning substrate produced by the dual beam multi-exposure technique. Figure 4.16 (left) depicts a photonic crystal pattern; this pattern was formed by vertical crossed line grating using two time exposures, with the distance between the two grating lines being $2 \mu \mathrm{m}$. Figure 4.16 (right) shows a 12 -fold rotational symmetry quasi-crystal pattern using six equal time exposures with the sample rotated $30^{\circ}$ after each exposure. 


\section{Chapter 5}

\section{Results and Discussion}

\subsection{Assembly of Colloidal Crystals with Non-Template}

\subsubsection{Proof of Laser Trapping and Manipulation}

The method described in this thesis is based on the self-arranging of particles in stage I by screened Coulomb repulsion and Brownian motion under the gradient force and scattering force of laser tweezers. The capillary force occurs during stage II and stage III completes the colloidal crystal complex fabrication process. Many factors contribute to the synthesis of colloidal photonic crystals. The principle of the selfarrangement of particles under a focused laser beam of laser tweezers is described as follows: first, a laser beam is used to gather the particles to the required area, vertical to the bottom surface of the chamber. Second, within that area the particles around the focused laser beam are gathered by the gradient force. Under the action of the laser trap, the particles in the illuminated area will be pushed to the surface of the chamber bottom. Third, the particles are packed together by the confinement of the bottom of the chamber and the gradient force and scattering force of the laser tweezers. The particles are gradually close-packed together as the system energy stabilizes. Fourth, after the processes of stages II and III, the particles finally come into contact with each other to form a close-packed colloidal photonic crystal. 
As the laser passes through the objective lens, it is focused to a diffraction limited elliptic spot. The function of the laser is demonstrated through the displacement of particles under the manipulation of the laser tweezers. Figure 5.1 illustrates the trapped particle moving from one position to the top of another particle under the laser tweezers.
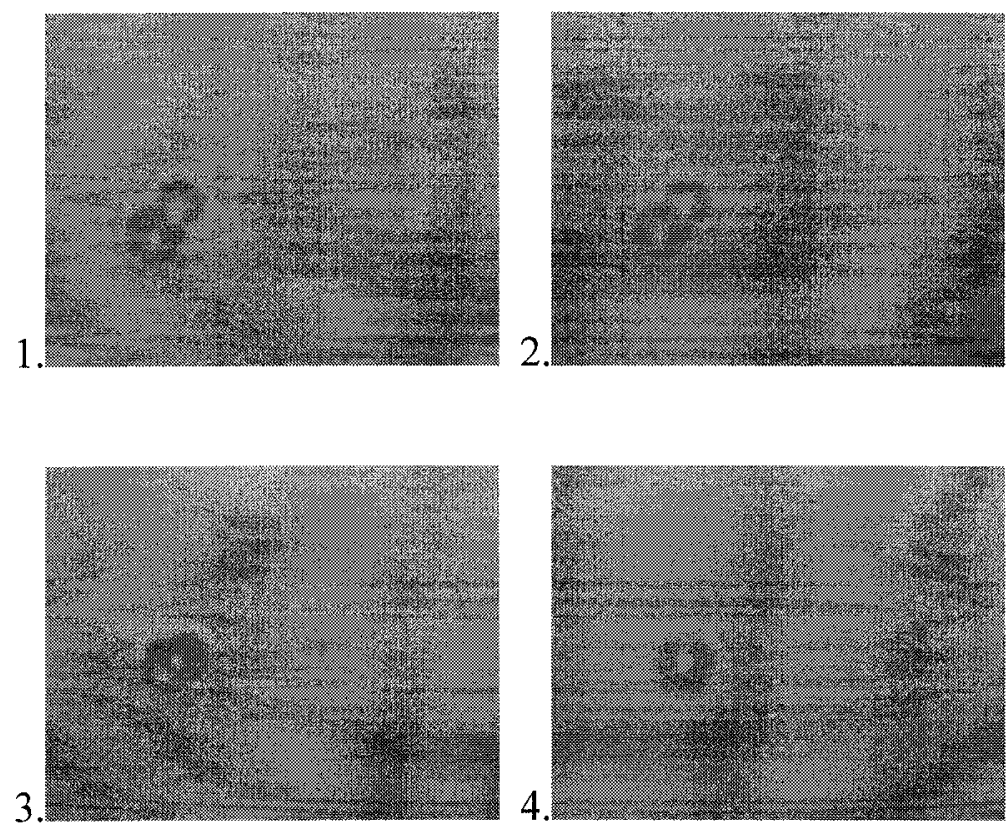

Figure 5.1 Illustration of particle displacement under the laser tweezers.

\subsubsection{Assembly Using Laser Tweezers}

Figure 5.2 shows the process of forming a 2D monolayer of colloidal crystals with a diameter of $2 \mu m$ under the action of laser tweezers. In the figure, the bright area around the center particle is the illuminated area of the focused laser beam. The particles are moved into the focused laser beam, gathered to the center by the trapping force, and self- 
arranged into the close-packed hexagonal lattice structure. Although the particles in the solvent are out of contact with each other due to Brownian motion, the particles remain close-packed as long as the forward laser beam is present.

1.

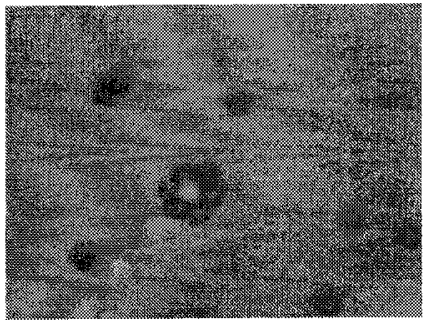

2.

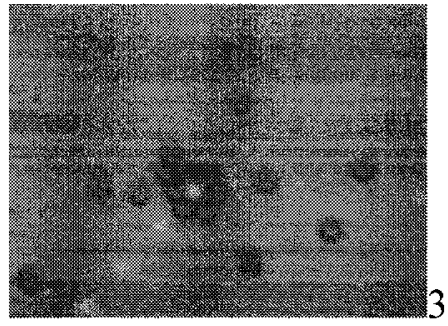

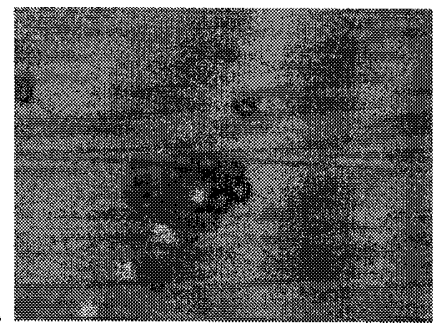

Figure 5.2 Process of forming a 2D monolayer of polystyrene colloidal crystals with a diameter of $2 \mu \mathrm{m}$ under the action of laser tweezers on a flat glass substrate.

\subsubsection{Results and Discussion of Assembly of 2D Colloidal Crystal}

From the band structure of a typical inverse-opal crystal in the photonic crystal theory described in Chapter 2, we know that the FCC inverse-opal structure with silicon matrix materials possesses a pseudo-gap between the fourth and fifth bands around a center frequency $\tilde{\omega}=0.524$ and a complete $4.25 \%$ band gap between the eighth and ninth bands with a center frequency of $\tilde{\omega} \approx 0.794$, as shown in Figure 2.5 DOS for a close-packed FCC lattice of air spheres in silicon $(\varepsilon=11.9)$ [10]. As the latter band gap lies at a higher-frequency, it is more sensitive to disorder effects in the crystal lattice. This band gap is narrow and a disorder magnitude as small as two percent of the lattice constant would lead to the closing of the band gap even in the 
presence of a very high refractive index contrast. The defects in the initial colloidal opal template will be directly imprinted in the final inverted opals. Therefore, the high requirements of the band gap impose a severe restriction on experimental efforts to control crystal uniformity [11].

As indicated in Section 5.1.2, the highly focused laser beam is used to manipulate and position particles around a specific area. The trapping process reassembles the particles in a monolayer of colloidal crystals deposited on the glass surface such that they are more evenly distributed. While the water solvent slowly evaporates, a 2D monolayer of polystyrene crystals is formed in a large area on a glass surface. Figure 5.3 shows some typical samples of 2D polystyrene colloidal crystals formed using the process mentioned above with a diameter of $2 \mu \mathrm{m}$ polystyrene colloidal particles with no laser beam present in the end. The pictures were randomly selected from different areas.

The optical images were captured by the laser tweezers with a $20 \mathrm{X}$ objective lens. In the optical images of the 2D colloidal crystals in Figure 5.3, the hexagonal structure of the crystal lattice is easily seen. Two kinds of defects are included: line type, corresponding to the crystal array shift along a line; and point type, which is usually due to the vacancy of a particle. The actual size of the image of the sample is $88.0 \times 66.0$ $\mu m^{2}$. 

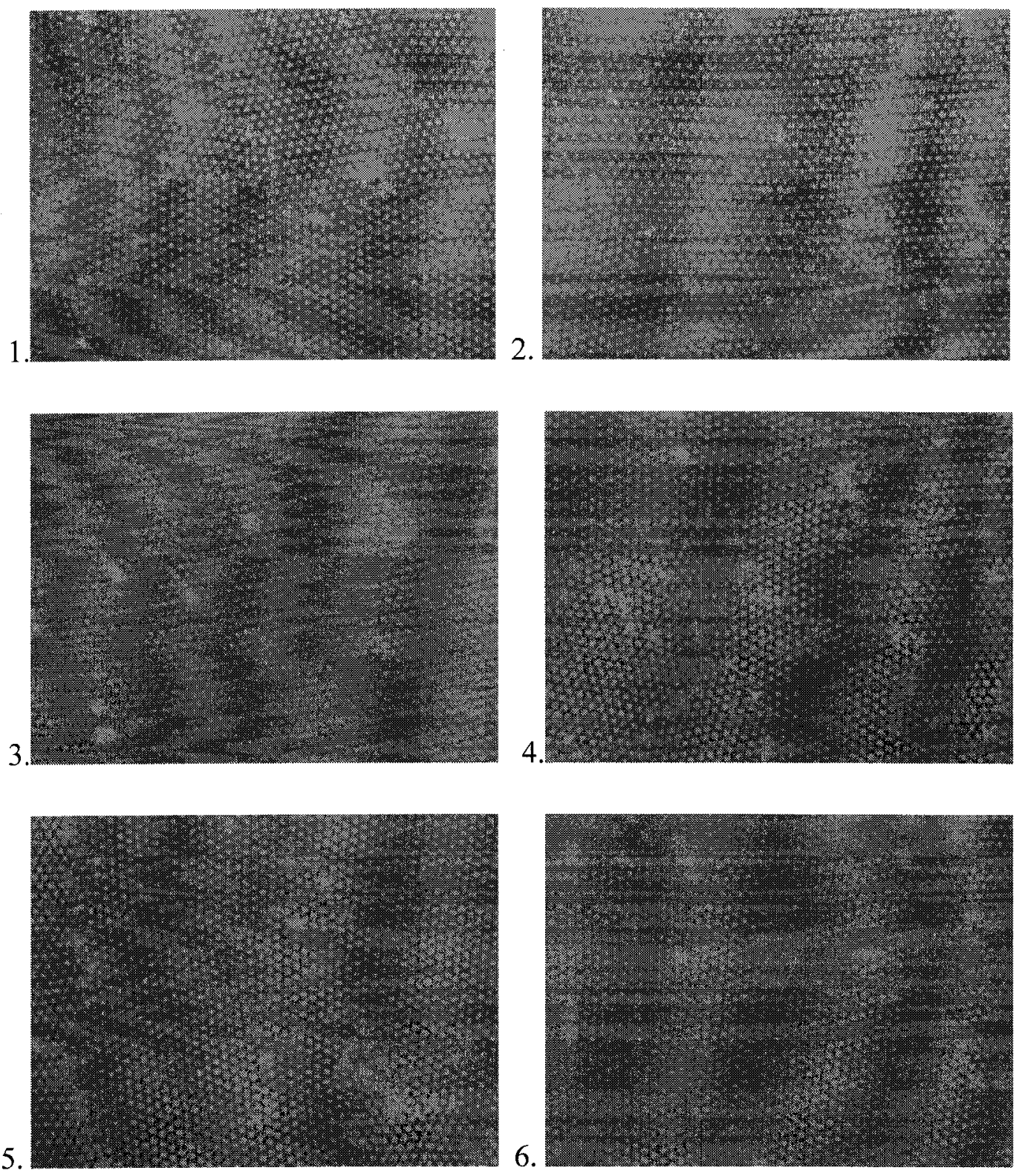

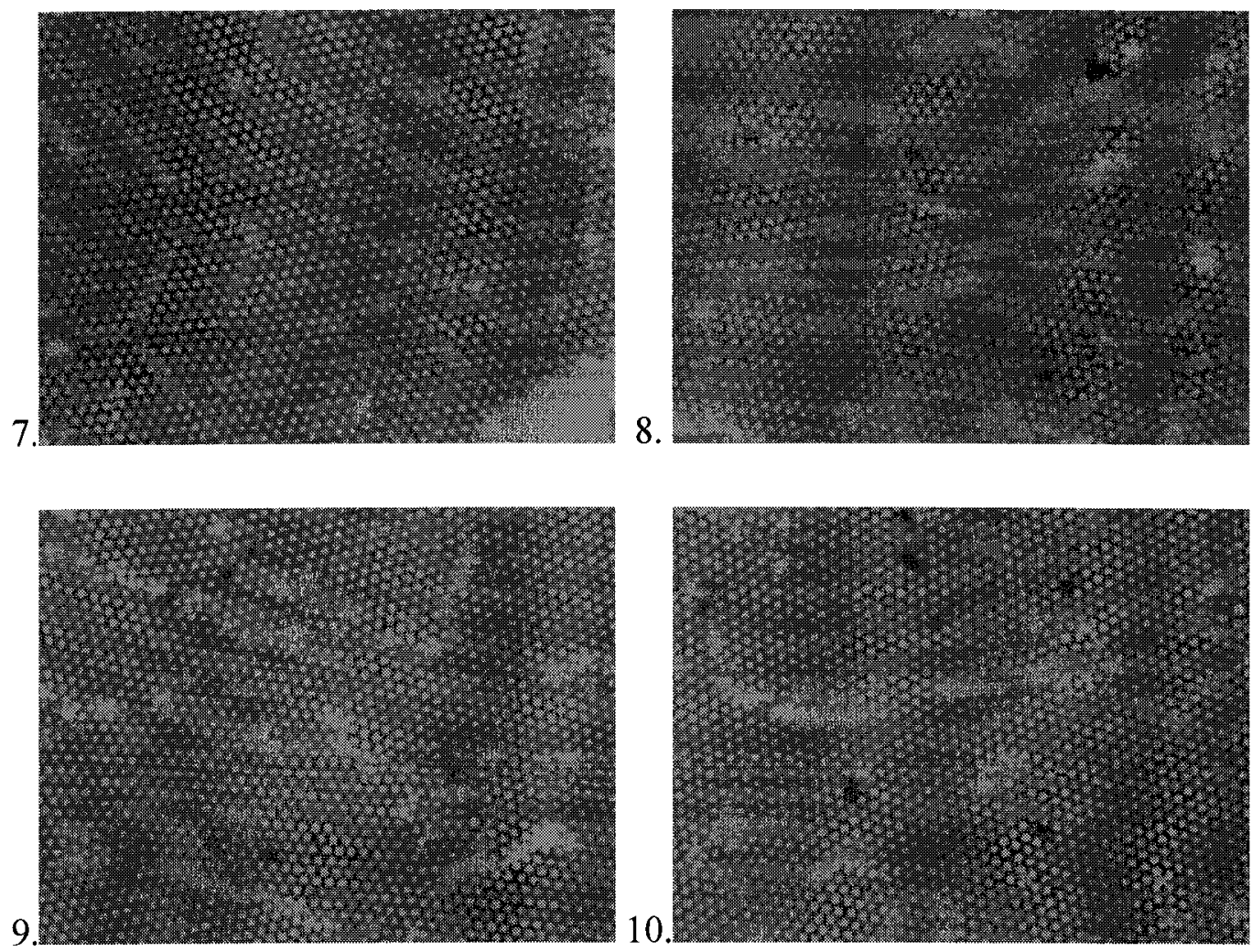

Figure 5.3 Optical microscopic images of the 2D colloidal crystal sample with an actual size of $88.0 \times 66.0 \mu \mathrm{m}^{2}$.

Table 5-1 lists the statistical results of defect density for 2D monolayer colloidal crystals. From the table, we can see that the average defect density is $0.0047 \mu \mathrm{m}^{-2}$, and that the sample defect density is nearly uniformly distributed. From Figures $5.3-6$, the maximum size of $40 \times 27 \mu \mathrm{m}^{2} 2 \mathrm{D}$ monolayer colloidal crystals without defects can be obtained. 
Table 5.1 Statistical Results of 2D Monolayer Colloidal Crystals for Fig.5.2

\begin{tabular}{|l|l|l|l|}
\hline No. of Figures & No. of Defects & Actual Size $\left(\mu \mathrm{m}^{2}\right)$ & Defect Density $\left(1 / \mu \mathrm{m}^{2}\right)$ \\
\hline 1 & 21 & $88.0 \times 66.0$ & 0.0036 \\
\hline 2 & 29 & $88.0 \times 66.0$ & 0.0050 \\
\hline 3 & 26 & $88.0 \times 66.0$ & 0.0045 \\
\hline 4 & 30 & $88.0 \times 66.0$ & 0.0052 \\
\hline 5 & 32 & $88.0 \times 66.0$ & 0.0055 \\
\hline 6 & 33 & $88.0 \times 66.0$ & 0.0057 \\
\hline 7 & 29 & $88.0 \times 66.0$ & 0.0050 \\
\hline 8 & 25 & $88.0 \times 66.0$ & 0.0043 \\
\hline 9 & 20 & $88.0 \times 66.0$ & 0.0034 \\
\hline 10 & 26 & $88.0 \times 66.0$ & 0.0045 \\
\hline Average & 27.1 & $88.0 \times 66.0$ & 0.0047 \\
\hline
\end{tabular}

Compared with self-assembly without the assistance of laser tweezers, the proposed method shows significant improvements in fabricating 2D monolayer colloidal crystals. Without the action of laser tweezers, the self-organization of particles occurs through capillary forces. This is a result of the slow movement of particles, which in turn form the suspension migrating into the liquid thin film at the drying front and aggregate into multiple layers over the colloid assembly pattern driven by capillary forces. Many factors, such as surface topography, influence the particles, and the form of the colloidal crystals is not predictable (see Figure 5.4 below). Therefore, the fabrication of monolayer 2D colloidal crystals is very difficult to control. Instead, using the proposed method, the particles can be gathered in a specific area and distributed more evenly under the influence of the tapping force and scattering force exerted by the laser tweezers. Large area monolayer 2D colloidal crystals are therefore more easily obtained. 


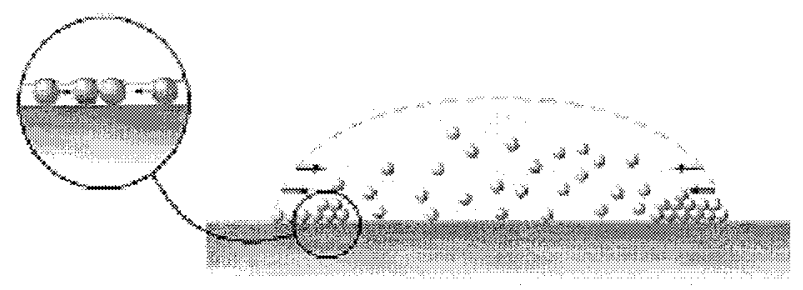

Figure 5.4 Schematic representation of a droplet of a colloidal suspension drying slowly on flat wall [22].

Three other typical 2D monolayer colloidal crystals that were fabricated are introduced below:

First, as we focus the laser beam to one point of the bottom of the glass substrate, the particles are gathered and close-packed together, and after the water has evaporated, a small area 2D monolayer will emerge. This kind of colloidal crystal is shown in Figures 5.5-1 and 5.5-2. The defect density of the colloidal crystals in Figure 5.5-1 is $0.0031 \mu \mathrm{m}^{-2}$, and the defect density of the colloidal crystals in Figure 5.5-2 is 0 . We may conclude that the defect density of this kind of small area colloidal crystal is lower than the average defect density of large area 2D colloidal crystals. 

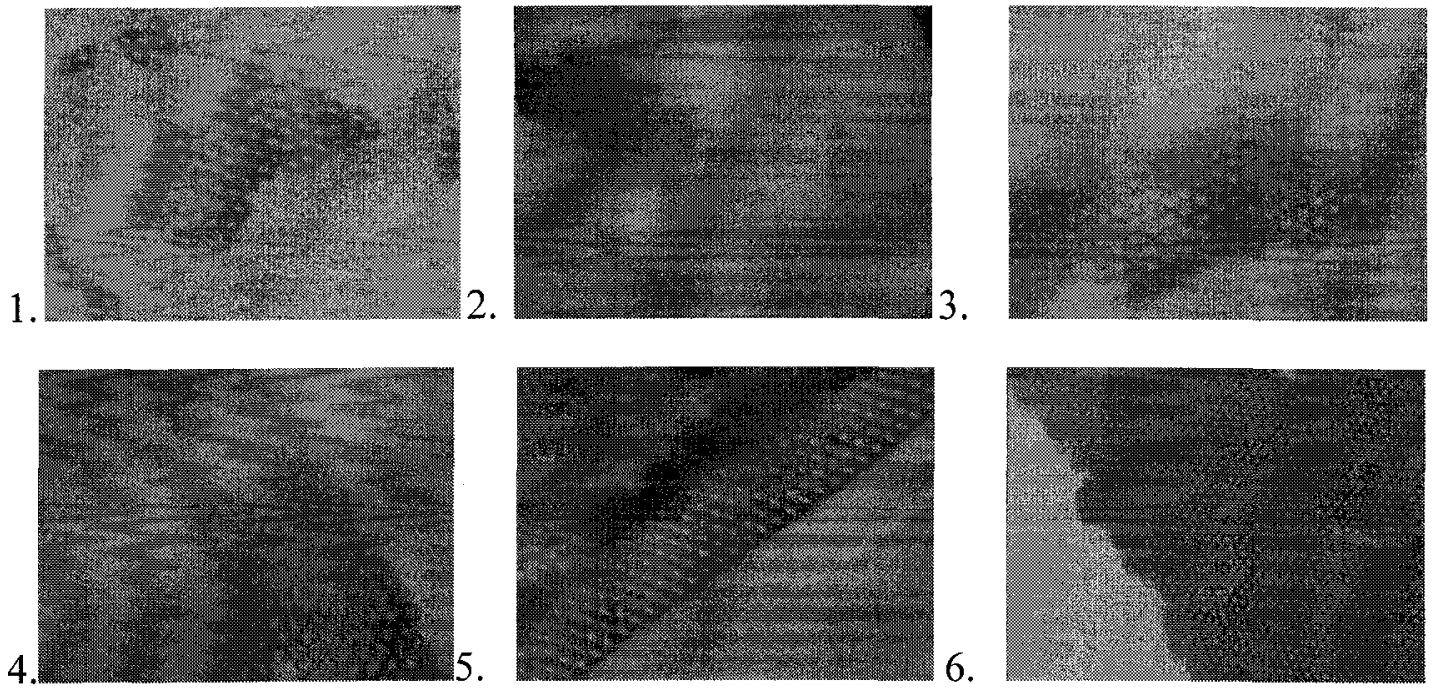

Figure 5.5 Typical close-packed 2D colloidal

photonic crystals obtained using laser tweezers.

Second, as we move the laser beam back and forth along the bottom of the glass substrate, the particles are gathered and close-packed together to form stripeshaped 2D monolayer colloidal crystals. This kind of colloidal crystal is shown in Figures 5.5-3 and 5.5-4. The defect density of the colloidal crystals in Figure 5.5-3 is $0.0025 \mu \mathrm{m}^{-2}$, and in Figure 5.5-4 it is 0.0038 . All of these are below the average defect density for large area 2D colloidal crystals.

Third, we used the laser beam to gather particles along the wall of the substrate. The particles were fabricated along the wall area. This kind of colloidal crystal is shown in Figures 5.5-5 and 5.5-6. The defect density of the colloidal crystals in Figure 5.5-5 is $0.0017 \mu \mathrm{m}^{-2}$, and in Figure 5.5-6 the defect density is $0.0023 \mu \mathrm{m}^{-2}$. With the help of the wall, the defect density decreases $0.0019 \mu^{-2}$, a considerable 
reduction. The typical results for density are summarized in Table 5-2, the average defect density being around $0.0022 \mu \mathrm{m}^{-2}$.

Table 5.2 Typical Statistical Results of 2D Monolayer Colloidal Crystals for Fig.5.6

\begin{tabular}{|l|l|l|l|}
\hline No. of Figures & No. of Defects & Actual Size $\left(\mu m^{2}\right)$ & Defect Density $\left(1 / \mu m^{2}\right)$ \\
\hline 1 & 1 & 320 & 0.0031 \\
\hline 2 & 0 & 136 & 0.0000 \\
\hline 3 & 1 & 400 & 0.0025 \\
\hline 4 & 2 & 520 & 0.0038 \\
\hline 5 & 1 & 580 & 0.0017 \\
\hline 6 & 12 & 5270 & 0.0022 \\
\hline
\end{tabular}

From the process of forming a 2D monolayer of polystyrene particles with a diameter of $2 \mu \mathrm{m}$ under the action of the laser tweezers as shown in Figure 5.2, and the final close-packed colloidal photonic crystals shown in Figures 5.3 and 5.6, we can see that the method we proposed has three advantages:

First, using the laser tweezers, a fine close-packed triangular 2D monolayer photonic crystal can be formed in a specific area, under the gradient forces and scattering forces of the laser tweezers. This allows a small area colloidal crystal to act as an effective kernel for the growth of large area patterns. As a result, the defective density in periodicity can be lowered.

Second, the laser tweezers can be used to control the growth of colloidal photonic crystals based on a fine close-packed triangular lattice structure with the help of a wall. 
Large area colloidal photonic crystals can be formed under controlled direction along the wall, and the defect density can be lowered.

Third, by means of this method, the polystyrene particles are self-organized into a close-packed lattice structure. To confirm this, the $2 \mathrm{D}$ monolayer of particles as shown in Figure 5.6 is clearly observed in the SEM picture.

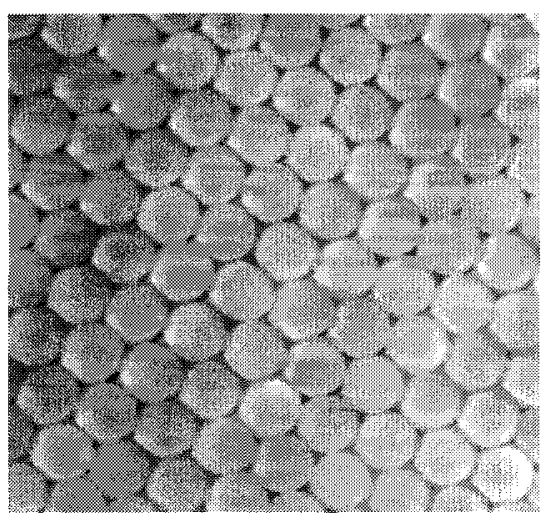

Figure 5.6 Scanning electron micrograph (SEM) of 2D monolayer colloidal crystals with $2 \mu \mathrm{m}$ polystyrene particles using laser tweezers.

\subsection{Assembly of 2D Colloidal Crystals with Template}

\subsubsection{Assembly of 2D Colloidal Crystals with Cylindrical Cavity Template}

This section discusses some useful results concerning the manipulation of colloidal particles using the laser tweezers on a specific patterned template, particularly on cylindrical cavity templates. The template is one kind of patterned substrate used to direct the growth of colloidal crystals. In this research, UV-light photolithography was used to build the cylindrical cavity and grid V-grooved template. 
Table 5.3 Control of the Self-Assembled Particles under Cylindrical Cavity Templates [23].

\begin{tabular}{|c|c|c|}
\hline Geometric shape of the template & Structures of the chuster & D/d \\
\hline \multirow{6}{*}{$+D+$} & & $1.00-2.00$ \\
\hline & & $2,00-215$ \\
\hline & & $2.15-2.41$ \\
\hline & & 2.41 .2 .70 \\
\hline & & $2.70-3.00$ \\
\hline & & $3,00-3,30$ \\
\hline
\end{tabular}

The need to confine the spherical colloids to the cylindrical cavity arises from the relationship between the diameter of the cylindrical cavity and the diameter of the spherical colloids. This is shown in Table 5.3. By using the proposed method with a highly focused laser beam, we use polystyrene particles to assemble more complex aggregates as shown in Figure 5.7. 
1.

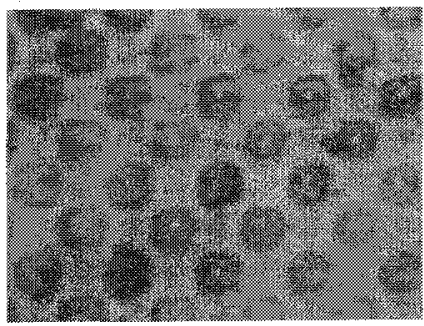

2.

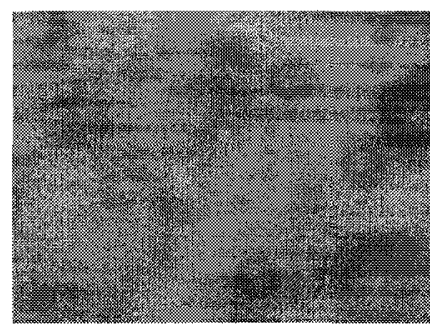

3.

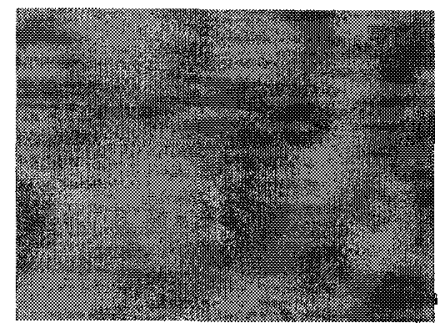

5 .

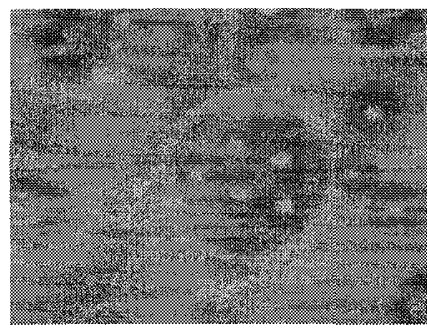

6.

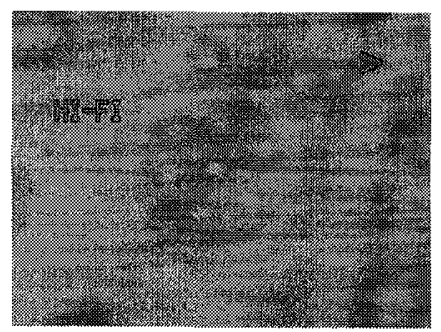

8.

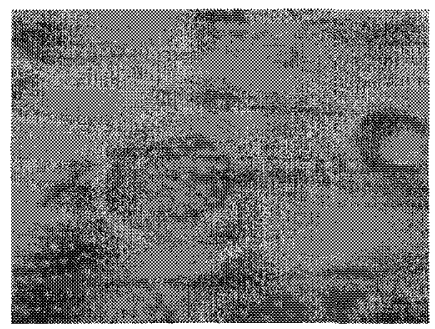

9.

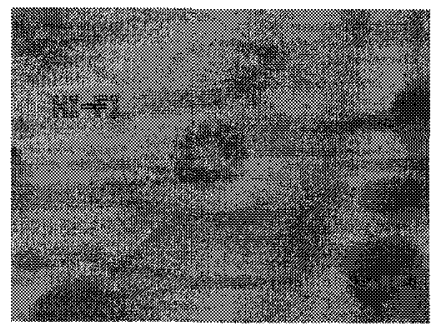

Figure 5.7 Video images of 2D arrays of colloidal aggregates assembled under the confinement of temples etched in the photoresist using laser tweezers.

In Figure 5.7-1, $2 \mu \mathrm{m}$ polystyrene beads were delivered in square distributed cylindrical cavities by using laser tweezers to move a polystyrene particle to each cavity, one at a time. Figures 5.7-2 to 5.7-9 were obtained using laser tweezers to focus on one fixed cavity, and the polystyrene particles ( $2 \mu \mathrm{m}$ polystyrene beads) were trapped together by carefully manipulating the laser beam. In Figure 5.7-2, a 2D array of three particles formed in a triangle, while in Figures 5.7-3 through 5.7-7, 2D arrays 
of four, five, six, seven and eight particles, respectively, were formed with $2 \mu \mathrm{m}$ polystyrene beads. In Figure 5.7-8, a 2D array of nineteen particles formed, and in Figure 5.7-9, a 2D ring of ten particles formed, both from $2 \mu \mathrm{m}$ polystyrene beads.

From these results, we can see that the laser tweezers enjoys a tremendous advantage in fabricating small area colloidal crystals without touching the polystyrene beads. By carefully manipulating the laser beam, defects in small area colloidal crystals can be avoided.

\subsubsection{Assembly of 2D Photonic Crystals with Grid Template}

1. Results:

Figure 5.8 shows the process of self-organizing the 2D monolayer of polystyrene photonic crystals with a diameter of $2 \mu \mathrm{m}$ under the action of laser tweezers on a patterned template. The patterned template was produced using the dual beam multiple exposure technique described in Section 4.6. The distance between two rows was $2 \mu \mathrm{m}$. The particles were gathered by gradient forces and scattering forces and numbered from several particles to tens of particles.

Initially, water was added to the chamber, and then six to eight drops of solution containing polystyrene particles were introduced into the water. Finally, a laser beam was focused to one fixed point in the patterned template. The laser beam trapped 7 particles, as shown in Figure 5.8-1, in which the location of the laser beam is indicated by an arrow. The particles were stabilized along the groove, and more particles were gathered along the groove while at the same time other particles accumulated in other 
grooves, forcing the colloidal particles to close-pack together while forming a larger area. The process from Figure 5.8-2 to Figure 5.8-3 lasted about 30 minutes.

1.
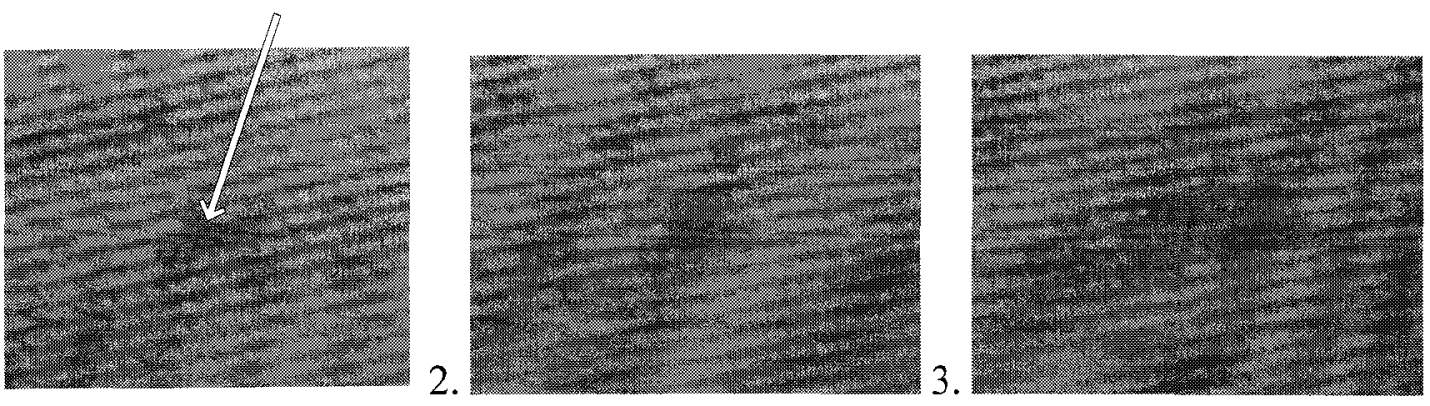

Figure 5.8 Process of self-organizing the 2D monolayer of polystyrene photonic crystals with a diameter of $2 \mu \mathrm{m}$ under the action of laser tweezers on patterned template.

Using this method, we obtained a greater diversity of form in the colloidal crystals on the square 2D grating template. These are shown in Figure 5.9. In Figure 5.9-1, 2x2 square colloidal crystals were formed. In Figure 5.9-2 3×3 square colloidal crystals were gathered. In Figure 5.9-3, a 4x3 rectangular form was obtained. In Figures 5.9-4 to 5.9-6, large area colloidal crystals were formed under the influence of the laser tweezers. 

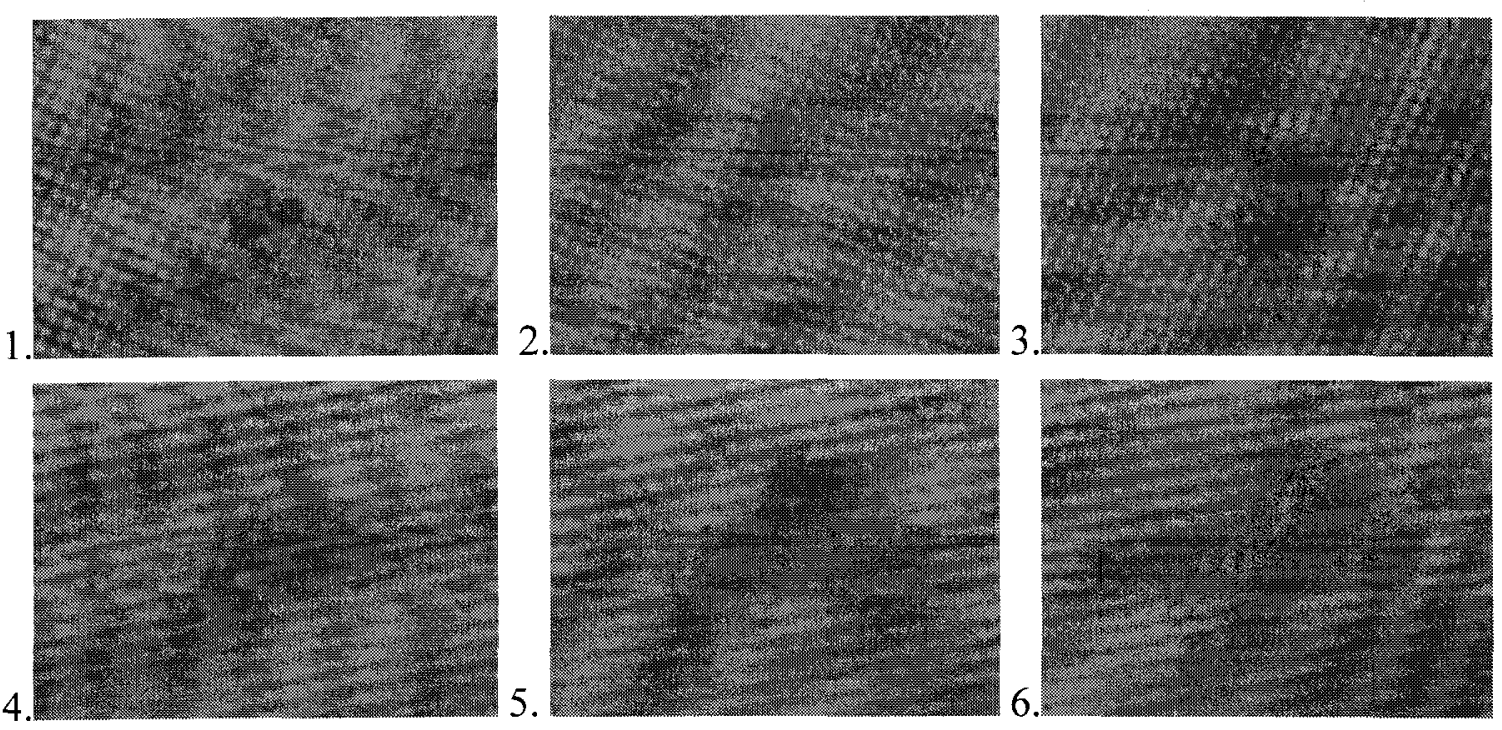

Figure 5.9 Typical colloidal crystals based on the square 2D grating template.

\section{Discussion:}

From Figure 5.9, we can see that the particles were arranged under the laser tweezers, and that they were not packed freely in a hexagonal lattice structure as they were with the flat wall set-up. In the patterned template, the particles are confined to the grid cavities, and the square symmetry of the template was transferred to the colloidal crystal and was maintained throughout its growth. The distance between two rows is $2 \mu m$, and the direction of the cavities led to the growth of the colloidal crystals. So, the lattice structure of the $2 \mathrm{D}$ template controls the lattice structure of the colloidal crystals. When using the flat wall, most particles are packed together in a close-packed hexagonal form or in a triangular form in the highest packing density. However, in the growth of colloidal crystals using the $2 \mathrm{D}$ patterned template, through controlling the space distance 
of the grooves, colloidal crystals with a lower packing density lattice structure can be obtained.

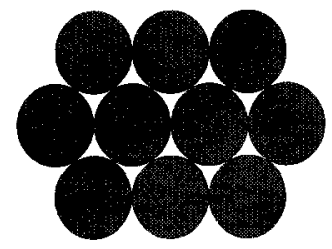

a.

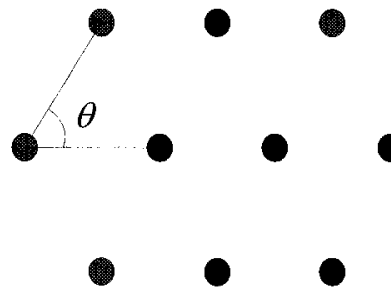

b.

Figure 5.10 a. Particle arrangement b. Lattice geometry.

From Figure $5.10 \mathrm{~b}$, with the angle $\theta=60^{\circ}$, the particles are arranged in a triangular structure. With an angle $\theta=90^{\circ}$, the particle arrangement results in a square structure. Using the pattern template and changing the angle of $\theta$, different arrangement structures may be obtained using laser tweezers. Therefore, the size of the particles and the size and shape of the template determines the growth pattern of the colloidal crystals.

The self-organization of colloidal particles in colloidal suspension and the capillary forces generated during the drying of the suspension are the main techniques in the growth of colloidal crystals. However, with laser tweezers to direct the selforganization process and position particles by drying on the patterned substrate template, there is no need for contact with the particles. If the high scanning speed of an acoustooptical deflector (AOD) is used, it is possible to create a time-shared multiple trap which can trap at least 100 particles simultaneously in a squared array. 
Compared with V-shaped grooves [24] in the substrate and pyramid-shaped microwells [25] [26], the cylindrical holes and 2D template fabricated using the dual laser beam multiple exposure technique is quick and simple. In the growth of colloidal crystals, it is possible to control the process using the laser tweezers to fabricate monolayer colloidal crystals in a specific area on the template and at the same time lower the incidence of defects as shown in Figure 5.9.

\subsection{Assembly of Quasi-Crystals with Template}

\subsubsection{Results:}

Figure 5.11 shows the process of self-organizing the 2D colloidal quasi-crystals with a diameter of $2 \mu \mathrm{m}$ polystyrene latex particles under the action of the laser tweezers on a patterned template.

First, water was added into the chamber with the patterned template bottom, and then 10 drops of solution containing polystyrene particles were introduced into the water. After a few hours, the polystyrene was observed at the bottom of the chamber, as shown in Figure 5.11-1. Then, we fixed the laser beam to one fixed point in the patterned template. Since the particles were not uniformly distributed in the cavity, the laser tweezers was used to move particles from other areas into the cavity. Figure 5.11-2 shows the particles distributed uniformly. With the evaporation of water and the particles falling to the bottom, more and more particles were gathered to the bottom, and in this process the laser tweezers was used to redistribute the particles uniformly. When the level of the water had nearly reached the bottom of the chamber, the particles were 
pushed to the groove of the template. The particles were gathered along the grooves, causing the colloidal particles to pack closely. A larger area view of the particles distributed along the quasi-crystal pattern is shown in Figure 5.11-3. The black area was distributed with polystyrene particles. The white area was the plain surface without etching. The process from Figure 5.11-1 to Figure 5.11-3 lasted about 30 minutes. Figure 5.11-3 showed that as the water began to withdraw to the cavity from the plain surface, the particles were pushed to the cavity and close-packed together along the cavity.

1.

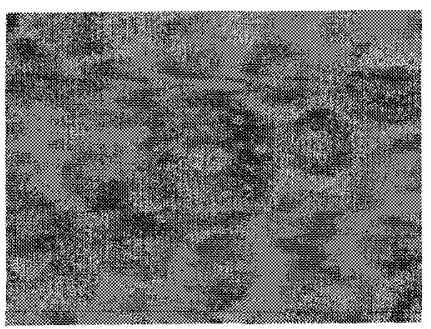

2.

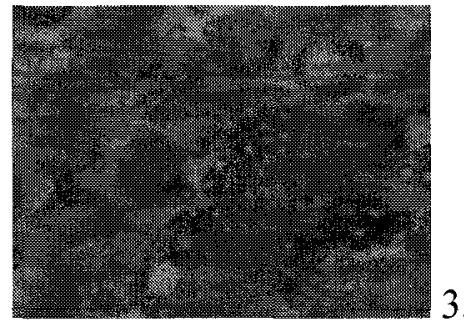

Figure 5.11 Process pictures showed the particles were close-packed together along the cavity of quasi-crystal pattern when the water evaporated.

Figure 5.12 shows pictures of the self-organizing 2D colloidal quasi-crystal sample with polystyrene latex particles with a diameter of $2 \mu m$ under the action of the laser tweezers on a patterned template with an actual size of $88.0 \times 66.0 \mu \mathrm{m}^{2}$. 

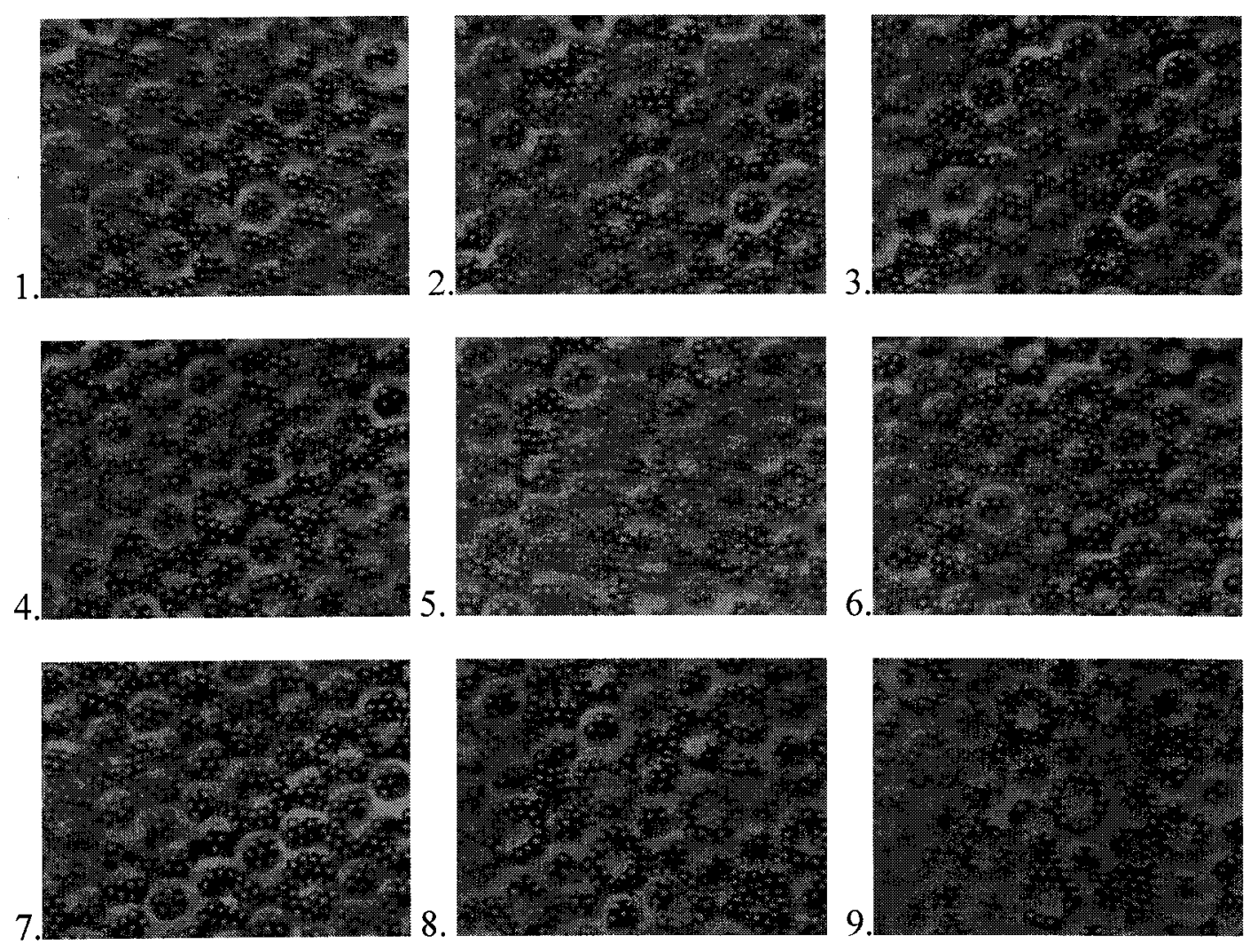

Figure 5.12 Optical microscopic images of the 2D quasi-crystal sample with an actual size of $88.0 \times 66.0 \mu \mathrm{m}^{2}$.

\subsubsection{Discussion:}

There are few differences in the growth of colloidal quasi-crystals. Laser tweezers are used to move the particles to the area just over the quasi-crystal template. Laser tweezers are also used to make the concentration of polystyrene particles distribute uniformly. The quantity of particles determines the final form and shape in the quasicrystal template. If the number of particles is not large enough, the quasi-crystal template 
will not fill fully, leading to uneven distribution. With quasi-crystals, the form of large area quasi-crystals is more important than with photonic crystals. This form will decide the band gap of the quasi-crystals. So, in the calculation of defective areas, only those sites which break the form of quasi-crystals can be included. Table 5.4 lists the statistical results of defect density for $2 \mathrm{D}$ colloidal quasi-crystals. From the table, we can see that the average defect density is $0.00060 \mu \mathrm{m}^{-2}$, and that the sample defect density is nearly uniformly distributed.

Table 5.4 Typical Statistical Results of 2D Colloidal Quasi-Crystals for Fig.5.14

\begin{tabular}{|l|l|l|l|}
\hline No. of Figures & No. of Defects & Actual Size $\left(\mu \mathrm{m}^{2}\right)$ & Defect Density $\left(1 / \mathrm{um}^{2}\right)$ \\
\hline 1 & 2 & $88.0 \times 66.0$ & 0.00035 \\
\hline 2 & 2 & $88.0 \times 66.0$ & 0.00035 \\
\hline 3 & 2 & $88.0 \times 66.0$ & 0.00035 \\
\hline 4 & 3 & $88.0 \times 66.0$ & 0.00051 \\
\hline 5 & 3 & $88.0 \times 66.0$ & 0.00051 \\
\hline 6 & 5 & $88.0 \times 66.0$ & 0.00086 \\
\hline 7 & 4 & $88.0 \times 66.0$ & 0.00069 \\
\hline 8 & 4 & $88.0 \times 66.0$ & 0.00069 \\
\hline 9 & 6 & $88.0 \times 66.0$ & 0.00103 \\
\hline Average & 3.5 & $88.0 \times 66.0$ & 0.00060 \\
\hline
\end{tabular}

The typical defect density is around $0.00060 \mu \mathrm{m}^{-2}$ as described in Table 5.4. Since the quasi-crystals are all fabricated in a large area, defects are inevitable. In this experiment, the laser tweezers was used simply to adjust the density of the particle distribution. We anticipate that using smaller particles would lower the defect density even more. 


\section{Chapter 6}

\section{Conclusion and Recommendations}

This thesis studied the fabrication of the monolayer of polystyrene colloidal crystals in a specific area under the gradient forces and scattering forces of the laser tweezers and the self-organization of polystyrene particles. The proposed method is based on a three stage model of colloidal synthesis. In stage I, the particles are selforganized under the action of the laser tweezers by screened Coulomb repulsion and Brownian motion. After stages II and III, the particles are self-assembled by capillary force. In this thesis, the growth of colloidal crystals using $2 \mu \mathrm{m}$ fine polystyrene particles under the laser tweezers has been considered, since the entire fabrication process needed to be confirmed by observations done by an optical imaging system with a microscope objective lens. Overall, the laser tweezers reduced the defect density from $0.0047 \mu m^{-2}$ to $0.0022 \mu \mathrm{m}^{-2}$. A larger defect-free monolayer of colloidal crystals in the triangular lattice structure was also obtained. Efforts in reducing defects with the help of a wall, cylindrical cavity and 2D square grating template were demonstrated experimentally. Furthermore, the fabrication of colloidal quasi-crystals based on a quasi-crystal template with low defect density was conducted with the assistance of laser tweezers.

On the basis of the experimental results, the significance of the proposed method is that it could be further developed to fabricate a 3D lattice structure of 
colloidal crystals by accumulating monolayers one above the other with the use of laser tweezers. Since localizing the growth of colloidal crystals in a specific area without touching them is possible with the assistance of laser tweezers, it should also be possible to control the fabrication of optical devices with colloidal crystals. The next step in research in this field should be focused on the fabrication of a 2D lattice structure of colloidal crystals using laser tweezers with smaller diameters of colloidal particles, such as $500 \mathrm{~nm}$ and $700 \mathrm{~nm}$, which correspond to $1.3 \mu \mathrm{m}$ and $1.5 \mu \mathrm{m}$, and to produce a photonic crystal template suitable for the communication wavelengths. 


\section{References}

[1] E. Yablonovitch, "Inhibited spontaneous emission in solid-state physics and electronics," Phys. Rev. Lett., vol. 58, pp. 2059-2062, 1987.

[2] S. John, "Strong localization of photons in certain disordered dielectric superlattices," Phys. Rev. Lett., vol. 58, pp. 2486-2489, 1987.

[3] J. D. Joannopoulus, R. D. Meade and J. D. Meade and J. N. Winn, Photonic Crystals; Modeling the flow of light, (Princeton University Press, 1995).

[4] I. I. Tarhan and G. H. Waltson, "Photonic band structure of fcc colloidal crystal," Phys. Rev. Lett., vol. 76, pp. 315-318, 1996.

[5] W. L. Vos, R. Sprik, A. van Blaaderen, A. Imhof, A. Lagendijk, and G. H. Wegdam, "Strong effects of photonic band structures on the diffraction of colloidal crystals," Phys. Rev. B, vol. 53, pp. 16231-16235, 1996.

[6] A. van Blaaderen, R. Ruel, and P. Wiltzius, "Templated-directed colloidal crystallization," Nature, vol. 385, pp. 321-324, 1997.

[7] Younan Xia, Byron Gates, and Sang Hyun Park, "Fabrication of Three-Dimensional Photonic Crystals for Use in the Spectral Region from Ultraviolet to Near-Infrared," Journal of Lightwave Technology, vol. 17, pp. 1956-1962, 1999.

[8] J. C. Maxwell, "A dynamical theory of the electromagnetic field." Philos. Trans. Roy. Coc. Lond. 155, 459, 1865.

[9] Steven G. Johnson and John D. Joannopoulos, Photonic Crystals, (Kluwer Academic Publishers, 2003). 
[10] Kurt Bush and Sajeev John, "Photonic band gap formation in certain self-organizing systems," Phys. Rev. E, vol. 58, pp. 3896-3908, 1998.

[11] Zhi-Yuan Li and Zhao-Qing Zhang, "Fragility of photonic band gaps in inverseopal photonic crystals," Phys. Rev. B 2000, 62, 1516.

[12] Robert C. Gauthier and Alexie Ivanov, "Production of Quasi- Crystal Template Patterns Using a Dual Beam Multiple Exposure Technique", Optics Express, 2004.

[13] Joydeep Dutta andHeinrich Hofmann "Self-organization of Colloidal nanoparticles", Encyclopedia of nanoscience and nanotechnology, Vol. X, pp. 1-23, 2003.

[14] Y. N. Xia, D. Qin, and Y. D. Yin, "Surface patterning and its application in wetting/dewetting studies," Current Opinion in Colloid \& Interface Science 6, pp. 54-64, 2001.

[15] Paul A. Steward, "Literature Review of Polymer Latex Film Formation and Particle Coalescence," http://www.initium.demon.co.uk/filmform.htm.

[16] A. Ashkin, "Acceleration and trapping of particles by radiant pressure," Phys. Rev. Lett., 24, pp. 156-159, 1970.

[17] A. Ashkin et al., "Observation of single-beam gradient force optical trap for dielectric particles," Opt. Lett., 11, pp. 288-290, 1986.

[18] Toshiaki Iwai, Katsuhiro Ishii, and Kuon Inoue, "Optical fabrications of ordered polystyrene particles using radiation pressure and self-organization," Proc. SPIE vol. 4598, pp. 94-100, 2001.

[19] Robert C. Gauthier et al., "Self-centering of a ball lens by laser trapping: fiberbal-fiber coupling analysis," Applied Optics, Vol. 42, No. 9, pp. 1610-1619, 2003. 
[20] A.Ashkin, "Forces of a single-beam gradient laser trap on a dielectric sphere in the ray optics regime," Biophys. J., vol. 61, pp. 569-582, 1992.

[21] Bahaa E. A. Saleh and Malvin Carl Teich, Fundamentals of Photonics, (John Wiley \& Sons, Inc., 1991).

[22] Joydeep Dutta and Heinrich Hofmann, "Self-Organization of Colloidal Nanoparticles," Encyclopedia of Nanoscience and Nanotechnology, vol. X, pp. 1-23, 2003.

[23] Y. Yin, Y. Lu, B. Gates, and Y. Xia, J. Am. Chem. Soc. 123, 8718, 2001.

[24] S. M. Yang and G. A. Ozin, "Opal chips: vectorial growth of colloidal crystal patterns inside silicon wafers," Chemical Communications, pp. 2507-2508, 2000.

[25] Y. D. Yin, Y. Lu, B.Gates, and Y. N. Xia, "Template-assisted self-assembly: A practical route to complex aggregates of monodispersed colloids with welldefined sizes, shapes, and structures," Journal of the American Chemical Society 123 , pp. 5298-5301, 1999.

[26] Geoffrey A. Ozin and San Ming Yang, "The race for the photonic chip: Colloidal crystal assembly in silicon wafers," Adv. Funct. Mater. No. 2, pp. 95-104, 2001. 


\section{Appendix}

\section{List of other parts for mounting laser tweezers:}

Cage assembly rods, THORLABS, ER3, ER2, ER4

$30 \mathrm{~mm}$ cage plates, THORLABS, CP02T

Mounting platform, THORLABS, B4C

Laser mount, THORLABS, S1LM9

Optical breadboard, THORLABS, 2" (50mm)

Posts, THORLABS, TR1, TR4, TR6

Post holders, THORLABS, PH

Table clamps, THORLABS, CL5

Bases, THORLABS,BA1S, BA1

Mounting post adapter, THORLABS, MA1

Angle bracket, THORLABS, AB90

V-clamp, THORLABS, PM1

Right angle clamps, THORLABS, RA90

Swivel clamps, THORLABS, SWC

Extension tubes, THORLABS, SM2E60

Lens tubes, THORLABS, SM2L20

SM-series adjustable focus, THORLABS, SM2V10

Stress free retaining ring, SM1 series, THORLABS, SM05

High intensity fiber light source, Dolan-Jenner 